\title{
Hafnium isotopic variations in East Atlantic intraplate volcanism
}

\author{
Jörg Geldmacher • Kaj Hoernle • Barry B. Hanan • \\ Janne Blichert-Toft $\cdot$ F. Hauff • James B. Gill • \\ Hans-Ulrich Schmincke
}

Received: 29 April 2010/Accepted: 1 September 2010/Published online: 19 September 2010

(C) The Author(s) 2010. This article is published with open access at Springerlink.com

\begin{abstract}
The broad belt of intraplate volcanism in the East Atlantic between $25^{\circ}$ and $37^{\circ} \mathrm{N}$ is proposed to have formed by two adjacent hotspot tracks (the Madeira and Canary tracks) that possess systematically different isotopic signatures reflecting different mantle source compositions. To test this model, Hf isotope ratios from volcanic rocks from all individual islands and all major seamounts are presented in this study. In comparison with published $\mathrm{Nd}$ isotope variations (6 $8 \mathrm{Nd}$ units), ${ }^{176} \mathrm{Hf} /{ }^{177} \mathrm{Hf}$ ratios span a much larger
\end{abstract}

Communicated by J. Hoefs.

Electronic supplementary material The online version of this article (doi:10.1007/s00410-010-0580-5) contains supplementary material, which is available to authorized users.

\section{J. Geldmacher $(\square)$}

Integrated Ocean Drilling Program, Texas A\&M University, 1000 Discovery Drive, College Station, TX 77845, USA

e-mail: geldmacher@iodp.tamu.edu

J. Geldmacher

Department of Geology and Geophysics, MS3115,

Texas A\&M University, College Station, TX 77843, USA

K. Hoernle · F. Hauff · H.-U. Schmincke

IFM-GEOMAR Leibniz-Institut für Meereswissenschaften,

Wischhofstr. 1-3, 24148 Kiel, Germany

B. B. Hanan

Department of Geological Sciences, San Diego State University, San Diego, CA 92182-1020, USA

\section{J. Blichert-Toft}

Laboratoire des Sciences de la Terre, Ecole Normale Supérieure de Lyon, 69364 Lyon, France

J. B. Gill

Earth \& Planetary Sciences Department, University of California

Santa Cruz, Santa Cruz, CA 95064, USA range (14 $\mathrm{eHf}$ units). Samples from the proposed Madeira hotspot track have the most radiogenic Hf isotopic compositions $\left({ }^{176} \mathrm{Hf} /{ }^{177} \mathrm{Hf}_{\mathrm{m}}\right.$ up to 0.283335$)$, extending across the entire field for central Atlantic MORB. They form a relatively narrow, elongated trend on the $\mathrm{Nd} \mathrm{vs}$. Hf isotope diagram (stretching over $>10 \varepsilon \mathrm{Hf}$ units) between a depleted N-MORB-like endmember and a moderately enriched composition located on, or slightly below, the Nd-Hf mantle array, which overlaps the proposed " $\mathrm{C}$ " mantle component of Hanan and Graham (1996). In contrast, all samples from the Canary hotspot track plot below the mantle array $\left({ }^{176} \mathrm{Hf} /{ }^{177} \mathrm{Hf}_{\mathrm{m}}=0.282943-0.283067\right)$ and form a much denser cluster with less compositional variation $(\sim 4 \varepsilon \mathrm{Hf}$ units). The cluster falls between (1) a low Hf isotope HIMUlike endmember, (2) a more depleted composition, and (3) the moderately enriched end of the Madeira trend. The new Hf isotope data confirm the general geochemical distinction of the Canary and Madeira domains in the East Atlantic. Both domains, however, seem to share a common, moderately enriched endmember that has " $\mathrm{C}$ "-like isotope compositions and is believed to represent subducted, $<1$-Ga-old oceanic lithosphere (oceanic crust and possibly minor sediment addition). The lower ${ }^{176} \mathrm{Hf} /{ }^{177} \mathrm{Hf}$ ratio of the enriched, HIMU-like Canary domain endmember indicates the contribution of oceanic lithosphere with somewhat older recycling ages of $\geq 1 \mathrm{Ga}$.

Keywords East Central Atlantic · Hf-isotopes · Hotspots · Mantle geochemistry · Madeira · Canary Islands

\section{Introduction}

The origin of intraplate volcanism, often associated with "hotspots", is a subject of ongoing debate (e.g. Anderson 
2001; Foulger and Natland 2003; Courtillot et al. 2003). Proposed surface hotspots generally fall above two widespread seismic anomalies, or superswells, in the lower mantle (e.g. Zhao 2003; Torsvik et al. 2006) that are interpreted as large-scale thermal upwellings or superplumes (e.g. Ritsema et al. 1999; Becker and Boschi 2002; Zhao 2003; Suetsugu et al. 2009). Nevertheless, establishing a direct link between surface volcanism and deepseated superplumes remains controversial. A possible compromise assigns the source region of hotspots to different mantle depths: for example, smaller upper mantle plumes ("secondary plumes") could originate from the top of the large superswells (Courtillot et al. 2003; Suetsugu et al. 2009) consistent with shallow mantle upwelling (e.g. Anderson 1998). One of the two recognized global lower mantle superswells is located beneath the African continent, the other beneath the Pacific Plate. The African Superswell extends roughly SE-NW from the southern Indian Ocean to the East Atlantic and can be traced upward $1,500 \mathrm{~km}$ from the core-mantle boundary ( $\mathrm{Ni}$ et al. 1999; Becker and Boschi 2002). A large concentration of hotspots, the Azores (e.g. Schilling 1975; White et al. 1976; Beier et al. 2008), Madeira (e.g. Mata et al. 1998; Geldmacher et al. 2000; Geldmacher and Hoernle 2000), Canary (e.g. Morgan 1972; Hoernle et al. 1991; Hoernle and Schmincke 1993; Geldmacher et al. 2001, 2005), and Cape Verde (e.g., Holm et al. 2006) can be found along the fringe of the superswell, on seafloor at the eastern edge of the African Plate. Recent seismic tomographic models indicate a low velocity anomaly in the upper mantle beneath the Canary Islands that merges with the Azores and Cape Verde anomalies at depths of 1,450-1,900 km before connecting to the African superswell in the deep mantle (Montelli et al. 2006).

The present $\mathrm{Hf}$ isotope study focuses on the area between $27^{\circ}$ and $36^{\circ} \mathrm{N}$, where several old and recently active intraplate volcanic island and seamount groups can be found, including the Canary, Madeira, and Selvagen Islands (Fig. 1). Although this is one of the geochemically best-investigated intraplate volcanic provinces on Earth (see Geldmacher et al. 2005 for overview), no Hf isotope ratios have yet been published for this region. Hafnium isotope ratios are a powerful tool for investigating intraplate volcanism and plume models, particularly in an oceanic setting where seawater/hydrothermal alteration can affect the $\mathrm{Rb}-\mathrm{Sr}$ and $\mathrm{U}-\mathrm{Th}-\mathrm{Pb}$ isotope systems. Among the radiogenic isotope systems used as mantle source tracers, the $\mathrm{Sm}-\mathrm{Nd}$ and, even more so, the $\mathrm{Lu}-\mathrm{Hf}$ isotope systems show the most immobile behavior in chloride-controlled aqueous fluid environments both at low and at high temperatures (e.g. Stalder et al. 1998; Thompson et al. 2008), making it particularly useful for investigating marine volcanic rocks. Hafnium is almost absent in seawater (average concentration of $0.00067 \mathrm{nmol} / \mathrm{kg}$ in the East Atlantic between 40 and $4,100 \mathrm{~m}$ water depth $=1.2 \times$ $10^{-7} \mathrm{ppm}$; Rickli et al. 2009). Furthermore, fractionation of the Lu/Hf ratio during partial mantle melting (e.g. between melt and cpx or garnet) is larger than that of the Sm/Nd ratio (e.g. Blundy et al. 1998), resulting, over time, in greater time-integrated ${ }^{176} \mathrm{Hf} /{ }^{177} \mathrm{Hf}$ reservoir variations compared with ${ }^{143} \mathrm{Nd} /{ }^{144} \mathrm{Nd}$. Thus, the presence of pyroxenitic lithologies (as proposed for many OIB lavas) has a major impact on the $\mathrm{Nd}$ and $\mathrm{Hf}$ isotopic systems (Hirschmann and Stolper 1996). Lu and Hf are also strongly fractionated by the Earth's sedimentary cycles, where $\mathrm{Hf}$ is a major constituent in the resistant heavy mineral zircon. In contrast to the rare earth element (REE), $\mathrm{Lu}$ and the high field strength element $\mathrm{Hf}, \mathrm{Sm}$ and $\mathrm{Nd}$, two neighboring REEs, are not fractionated significantly from each other in sedimentary systems (e.g. Vervoort et al. 1999). The net effect is that $\mathrm{Hf}$ isotopes are a sensitive tracer for source variations in intraplate magmas, such as those resulting from the presence of recycled crustal material in the magma source (e.g. Blichert-Toft et al. 1999). To supplement the current geochemical data base for eastern North Atlantic intraplate volcanism and to reassess previously proposed regional hotspot models, we here present $\mathrm{Hf}$ isotope measurements for 61 samples from the Selvagen, Madeira and Canary Island groups, and from major seamounts in that region, including Ampère, Unicorn, Seine, Dacia, Conception, Nico, Essaouira (working name "Lars" in previous publications), and Last Minute, which vary in evolutionary stage and mantle source signature. $\mathrm{Sr}, \mathrm{Nd}$, and $\mathrm{Pb}$ isotope ratios have been published previously for these samples. In addition, we also present new $\mathrm{Sr}, \mathrm{Nd}$, and $\mathrm{Pb}$, together with their $\mathrm{Hf}$ isotope data, for samples from six Canary islands.

\section{Geological overview and summary of previous work}

Regional models for the origin of eastern Central-Atlantic intraplate volcanism can be grouped into non-plume models (e.g., Schmincke 1982; King and Anderson 1998; Anguita and Hernán 2000; King and Ritsema 2000), plume models (e.g., Burke and Wilson 1972; Duncan 1984; Hoernle et al. 1991; Hoernle and Schmincke 1993a, b; Hoernle 1998; Carracedo 1994; Mata et al. 1998; Widom et al. 1999; Geldmacher et al. 2000; Gurenko et al. 2006), or any combination of both (e.g. Carracedo et al. 1998; Geldmacher et al. 2005, 2006a; Schmincke and Sumita 2010). Plume models range from multiple narrow, individual hotspots (e.g. Hoernle et al. 1991; Hoernle and Schmincke 1993b; Geldmacher et al. 2000, 2001) to largescale regional upwellings and mega plumes (e.g. Hoernle et al. 1995; Merle et al. 2006, 2009). 


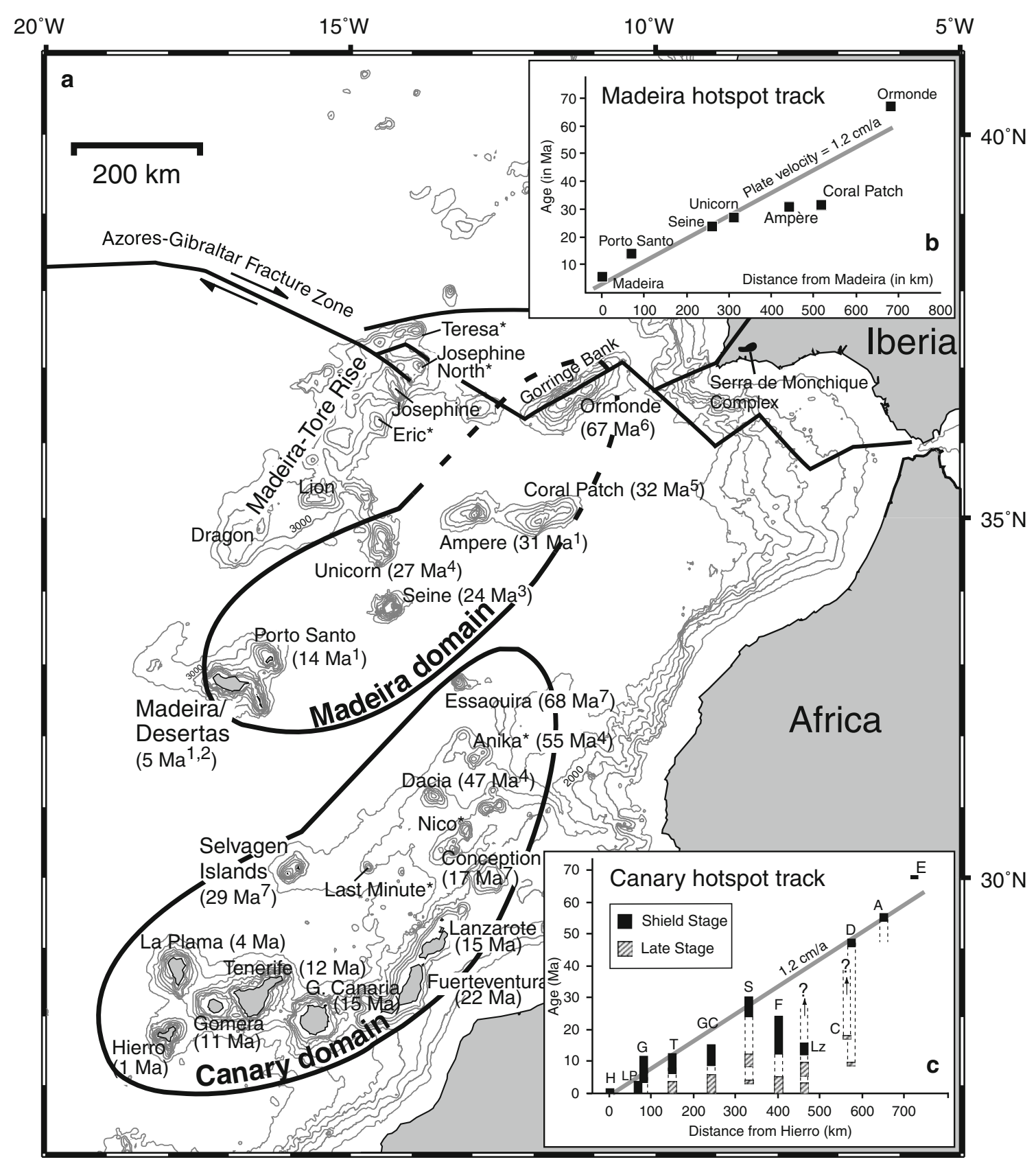

Fig. 1 a Bathymetric map of the central northeast Atlantic after Smith and Sandwell (1997) (only depths contours above 3,500 m are shown for clarity). Proposed Madeira and Canary domains are encircled. Asterisks (*) indicate working names for unnamed seamounts. Note that Essaouira Seamount was given the working name "Lars" in some previous publications. Ages in parenthesis are oldest available radiometric ages taken from (1) Geldmacher et al.

Based on plate tectonic kinematics and ${ }^{40} \mathrm{Ar} /{ }^{39} \mathrm{Ar}$ age dating, the regional volcanic pattern can be interpreted to reflect two parallel but independent hotspot tracks, Madeira and Canary (Morgan 1972; Duncan 1984; Geldmacher et al. 2001, 2005). The present location of the Madeira and Canary hotspots are considered to be at the southwestern ends of the tracks, at Madeira and Hierro Islands,
(2000); (2) Klügel et al. (2009); (3) Merle et al. (2009); (4) Geldmacher et al. (2005); (5) D'Oriano et al. (accepted); (6) Féraud et al. (1986); (7) Geldmacher et al. (2001). Canary Island ages from the literature (see Schmincke and Sumita 2010 for overview). b and c age versus distance relationship for islands and seamounts of the Madeira and Canary hotspot tracks (updated after Geldmacher et al. 2005)

respectively (e.g. Hoernle et al. 1991; Hoernle and Schmincke 1993a, b; Caracedo et al. 1998; Geldmacher et al. 2000, 2005) (see Fig. 1b, c). The existence of at least two distinct magma sources is further supported by distinct $\mathrm{Pb}$ and $\mathrm{Nd}$ isotope trends observed in volcanic rock samples from both hotspot chains (e.g., Geldmacher et al. 2001). Samples collected from islands and seamounts of 
the proposed Madeira hotspot track (Madeira, Desertas, Porto Santo Islands and Seine and Unicorn seamounts) tend to have slightly lower ${ }^{207} \mathrm{~Pb} /{ }^{204} \mathrm{~Pb}$ and higher ${ }^{143} \mathrm{Nd} /{ }^{144} \mathrm{Nd}$ for a given ${ }^{206} \mathrm{~Pb} /{ }^{204} \mathrm{~Pb}$ than samples from the proposed Canary hotspot track (Canary and Selvagen Islands, and Last Minute, Nico, Dacia, and Essaouira seamounts to the $\mathrm{NE})$ resulting in two distinct isotopic fields or "domains" on isotope plots (Fig. 2a, b). A notable exception is observed for the few available samples from the northern (oldest) end of the proposed Madeira hotspot track, Ampère and Ormonde Seamounts, which exhibit elevated ${ }^{207} \mathrm{~Pb} /{ }^{204} \mathrm{~Pb}$ and reduced ${ }^{143} \mathrm{Nd} /{ }^{144} \mathrm{Nd}$ compared to their ${ }^{206} \mathrm{~Pb} /{ }^{204} \mathrm{~Pb}$ ratios. A possible explanation could be the increasing influence of continental lithosphere (having a radiogenic isotope composition with high ${ }^{207} \mathrm{~Pb} /{ }^{204} \mathrm{~Pb}$ and low ${ }^{143} \mathrm{Nd} /{ }^{144} \mathrm{Nd}$ ) with decreasing distance of the hotspot track to the continental margin (Geldmacher and Hoernle 2000). This relationship becomes even clearer, if the volcanic massifs along the coast of SW Portugal (see Fig. 1a), possessing similar or higher ${ }^{207} \mathrm{~Pb} /{ }^{204} \mathrm{~Pb}$ and lower ${ }^{143} \mathrm{Nd} /{ }^{144} \mathrm{Nd}$ ratios (Bernard-Griffiths et al. 1997), are also considered as being part of the Madeira hotspot track (Geldmacher et al. 2000; Geldmacher and Hoernle 2000).

Another enigmatic feature that does not fit into a simple hotspot model is the Madeira-Tore Rise. Results of ${ }^{40} \mathrm{Ar} /{ }^{39} \mathrm{Ar}$ and $\mathrm{U} / \mathrm{Pb}$ age determinations, as well as paleontological constraints, recently revealed that this $\sim 1,000$ $\mathrm{km}$-long submarine ridge was initially formed in the Late Cretaceous (Geldmacher et al. 2006a; Merle et al. 2006). Based on plate tectonic reconstructions and gravity data, the Madeira-Tore Rise could have formed as an aseismic ridge (e.g., Peirce and Barton 1991) possibly by interaction of the westward-migrating Mid-Atlantic spreading center with the early Canary hotspot (Geldmacher et al. 2006a). According to plume-ridge interaction models in which a ridge crosses a hotspot (e.g., Schilling et al. 1985; Sleep 2002), aseismic ridges continue to form as long as the plume is near enough to the ridge for buoyant plume material to flow along the base of the lithosphere toward the ridge. If the migrating ridge becomes too distant to allow for this process to take place, a distinct new hotspot track forms on the new plate leaving a spatial gap of surface volcanism between the aseismic ridge and the onset of the new hotspot track. The onset of the Canary hotspot track on the African plate is proposed to be located near Essaouira seamount (Fig. 1) appearing at around $70 \mathrm{Ma}$ (Geldmacher et al. 2001, 2005, 2006a). This model is further supported by the isotopic composition of dredge samples from the Cretaceous base of the Madeira-Tore Rise (Lion and Teresa Seamounts at the southern and northern ends of the rise, respectively) plotting well within the Canary domain field (Fig. 2). Although only the measured $\mathrm{Pb}$ isotope ratios are plotted in Fig. 2 (to allow for better comparison of samples of different ages generated by evolving mantle sources, based on the assumption that the $\mathrm{U} / \mathrm{Pb}$ ratio is not significantly affected by the melting process), the age-corrected $\mathrm{Pb}$ isotope ratios of $92 \mathrm{Ma}$ old Teresa seamount samples $\left({ }^{206} \mathrm{~Pb} /{ }^{204} \mathrm{~Pb}_{\text {in }}=19.444-19.553\right.$, $\left.{ }^{207} \mathrm{~Pb} /{ }^{204} \mathrm{~Pb}_{\text {in }}=15.584-15.589\right)$ would still plot well within today's Canary domain. Likewise, the only available $\sim 75 \mathrm{Ma}$ old Lion seamount sample $\left({ }^{206} \mathrm{~Pb} /{ }^{204} \mathrm{~Pb}_{\text {in }}=\right.$ $\left.18.298,{ }^{207} \mathrm{~Pb} /{ }^{204} \mathrm{~Pb}_{\text {in }}=15.542\right)$ plots within the zero-age MORB field well above the Northern Hemisphere Reference Line (NHRL).

Interestingly, several younger (Miocene to recent) volcanic edifices seem to be superimposed on the Cretaceous base of the Madeira-Tore Rise, mainly at its northern end, where the rise is cut by the active Azores-Gibraltar-Fracture Zone fault system. The geochemical composition of these Miocene to recent lavas cannot be clearly attributed to either the Madeira or the Canary isotopic domain but seem to define a bimodal mixing line between ambient Central Atlantic MORB and a composition near the enriched end of the Canary domain field (e.g. ${ }^{206} \mathrm{~Pb} /{ }^{204} \mathrm{~Pb} \approx$ 23 and ${ }^{143} \mathrm{Nd} /{ }^{144} \mathrm{Nd} \approx 0.5128$, see Fig. 2) (Geldmacher et al. 2006a). Alternatively, these isotopically enriched samples (high $\mathrm{Pb}$ but low $\mathrm{Nd}$ isotope ratios) lie on an extension of the Madeira array. The few available isotope data from young Madeira-Tore Rise volcanism make it difficult to determine whether its enriched endmember should be attributed to the Canary or the Madeira domains, or whether it is a component distinct from both. This Miocene to recent volcanism at the northern Madeira-Tore Rise maybe caused by local extension resulting in shallow mantle decompression melting along and near the Azores Gibraltar Fracture zone. Such melts could mingle with trapped Canary domain material left from the time of the rise's formation or tap recent Madeira domain material, which could have spread out at the base of the lithosphere toward the rise. However, both explanations are not entirely satisfying since one location (Dragon Seamount, from which the only dated sample yielded a young ${ }^{40} \mathrm{Ar} /{ }^{39} \mathrm{Ar}$ plateau age of $3.94 \pm 0.30 \mathrm{Ma}$, Geldmacher et al. 2006a) lies at the southern end of the Madeira-Tore Rise (far away from any known active fault zone). The alternative model, attributing the origin of the entire Madeira-Tore Rise and the neighboring islands and seamounts of the Madeira domain to a long-lived thermal anomaly (mega plume) with a generally but broad southward-migrating, pulsating magma production below the lithosphere (Merle et al. 2006; Merle et al. 2009) would allow for an easier integration of this outlier sample from Dragon Seamount. Recent swath bathymetric mapping, however, supports the existence of local extension zones at the northern end of the MadeiraTore Rise (Zitellini et al. 2009).

Below, we will evaluate the new Hf isotope data in light of the hotspot models proposed above. 


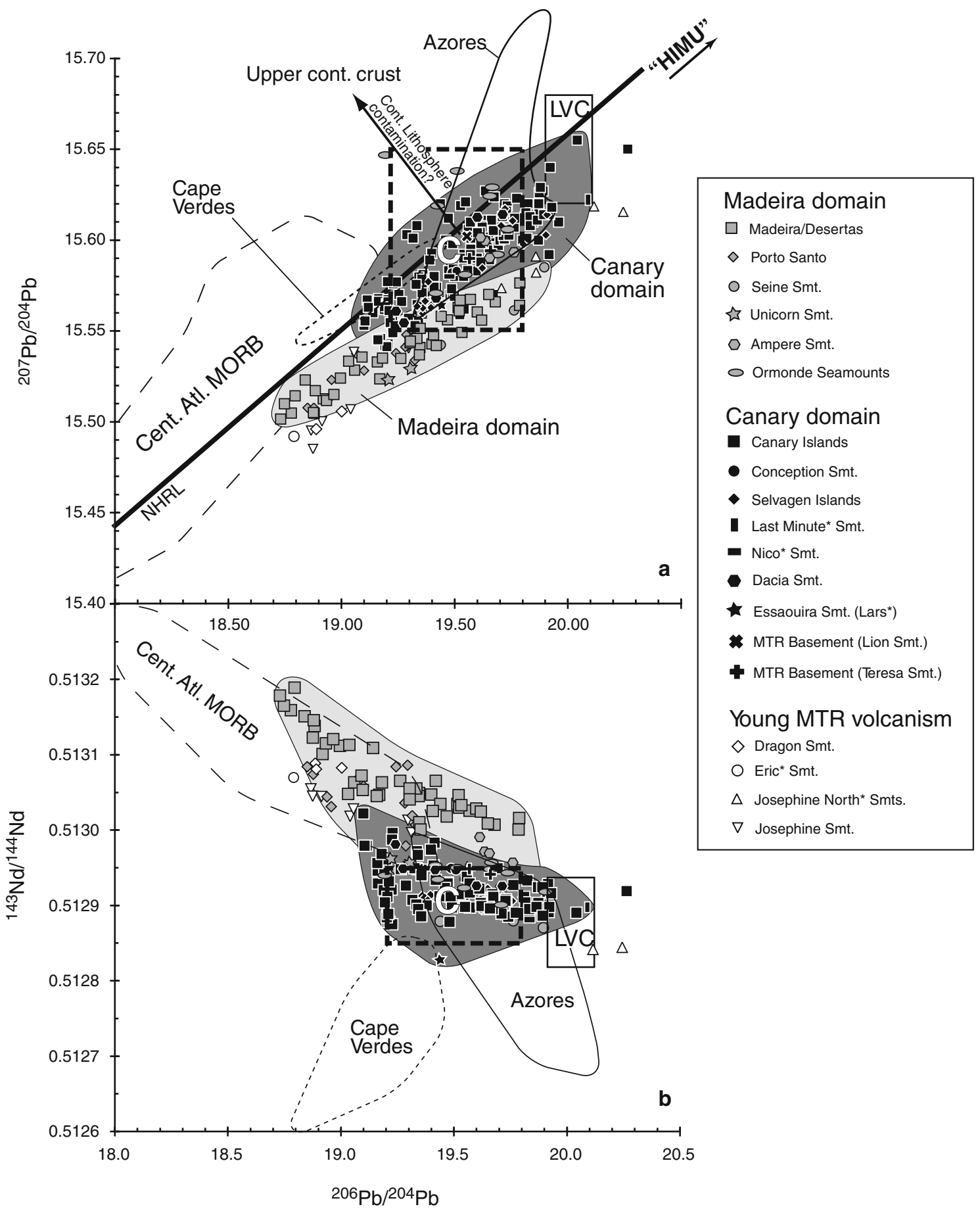

Fig. $2{ }^{207} \mathrm{~Pb} /{ }^{204} \mathrm{~Pb}$ (a) and ${ }^{143} \mathrm{Nd} /{ }^{144} \mathrm{Nd}$ (b) isotope variations versus ${ }^{206} \mathrm{~Pb} /{ }^{204} \mathrm{~Pb}$ of Canary and Madeira domain samples and young volcanic features on the Madeira-Tore Rise. Central Atlantic MORB data from $25^{\circ}$ to $34.9^{\circ} \mathrm{N}$ only (to avoid known melting anomalies) from the PetDB database (http://www.petdb.org/). Canary Island domain sources: Cousens et al. (1990); Hoernle and Tilton (1991); Hoernle et al. (1991); Geldmacher et al. (2001, 2005); Gurenko et al. (2006); and this study. Madeira domain data sources: Geldmacher and
Hoernle (2000); Geldmacher et al. (2005); Bernard-Griffith et al. (1997); Madeira-Tore Rise: Geldmacher et al. (2006a). Fields for Azores islands São Miguel and Pico are from Beier et al. (2007) and Elliot et al. (2007) and for the Cape Verde island Santiago from Barker et al. (2009) and Martins et al. (2010). Common mantle endmember "C" composition after Hanan and Graham (1996). LVC ("Low velocity component"), a previously proposed common enriched endmember, after Hoernle et al. (1995) 


\section{Sample selection and analytical techniques}

Sixty-one representative samples from all volcanic islands and major seamounts were selected for this study (Table 1). Most samples are of mafic, mainly basaltic composition, with Mg-numbers within or near the range of magmas in equilibrium with mantle olivine (68-75). No correlation, however, was found between $\mathrm{Mg}$-number and ${ }^{176} \mathrm{Hf} /{ }^{177} \mathrm{Hf}$ ratios in the present data set, implying that magma fractionation processes can be neglected. Majorand trace element concentrations, $\mathrm{Sr}, \mathrm{Nd}$, and $\mathrm{Pb}$ isotope ratios, and age information of all Madeira, Selvagen and seamount samples are reported in Geldmacher and Hoernle (2000), Geldmacher et al. $(2001,2005)$ and are compiled in Appendix 1. Corresponding $\mathrm{Sr}, \mathrm{Nd}$, and $\mathrm{Pb}$ isotope ratios of all Canary samples used in this study are given in Table 2.

Hafnium separation chemistry for all but three samples was carried out at San Diego State University using rock powders prepared from carefully hand-picked whole rock chips. For chemical separation, we followed the procedure of Blichert-Toft et al. (1997). About a quarter of the samples were measured at Ecole Normale Supérieure in Lyon using a first-generation "VG Plasma 54" multiple collector magnetic sector inductively coupled plasma mass spectrometer (MC-ICP-MS) in one consecutive measurement session. ${ }^{176} \mathrm{Hf} /{ }^{177} \mathrm{Hf}$ was normalized for instrumental mass bias relative to ${ }^{179} \mathrm{Hf} /{ }^{177} \mathrm{Hf}=0.7325$. The JMC-475 $\mathrm{Hf}$ standard was run repeatedly every $2-3$ samples and averaged $0.282169 \pm 17 \quad(n=8)$. The average in-run precision of sample ${ }^{176} \mathrm{Hf} /{ }^{177} \mathrm{Hf}$ is $0.000008(2 \sigma)$. The majority of the samples were measured on a second-generation "Nu Plasma HR" at San Diego State University with an average in-run precision of $0.000003(2 \sigma)$. An in-house standard (cross-calibrated to JMC-475) was measured every three samples to monitor machine performance. Five samples (three new samples from Seine and Unicorn Seamounts and two samples previously measured in Lyon) were chemically processed and measured at IFMGEOMAR in Kiel on an AXIOM MC-ICP-MS (see Geldmacher et al. 2006a for operating details and machine performance). To ensure compatibility of all data and to rule out any possible machine bias, all isotope ratios measured in Lyon, San Diego, and Kiel were normalized by standard bracketing relative to ${ }^{176} \mathrm{Hf} /{ }^{177} \mathrm{Hf}=0.282162$ for JMC-475. As shown in Table 1, normalized duplicate analyses of samples processed/measured in San Diego/ Lyon and Kiel (Ma 17 and Ma 88) are within analytical error of each other.

The $\mathrm{Sr}, \mathrm{Nd}$, and $\mathrm{Pb}$ isotope ratios of most Canary Island samples were measured at UC Santa Cruz on a VG54-30 TIMS using chemical separation techniques as described in Hoernle and Tilton (1991) (Table 2). Sr and $\mathrm{Nd}$ isotope ratios were fractionation-corrected to NBS $987\left({ }^{87} \mathrm{Sr} /{ }^{86} \mathrm{Sr}=0.710250\right)$ and to the La Jolla standard $\left({ }^{143} \mathrm{Nd} /{ }^{144} \mathrm{Nd}=0.511850\right)$. A subset of samples-indicated by asterisks - were measured in static multicollection mode by TIMS at IFM-GEOMAR (Kiel, Germany) on a ThermoFinnigan TRITON ( $\mathrm{Sr}-\mathrm{Nd})$ and MAT262 (Pb). Similar to the UCSC data, the IFM-GEOMAR data were normalized to these standards to allow for the best possible data consistency between the labs (although measurements of the standards, run together with the samples, were identical to the accepted values ${ }^{87} \mathrm{Sr} /{ }^{86} \mathrm{Sr}=0.710250 \pm$ $0.000001 \quad(n=2)$ for $\mathrm{NBS} 987$ and ${ }^{143} \mathrm{Nd} /{ }^{144} \mathrm{Nd}=$ $0.511850 \pm 0.000006(n=7)$. All $\mathrm{Pb}$ isotope ratios were externally fractionation-corrected by $0.115 \% / a m u$ derived from the normalization of measured NBS981 values to the NBS981 reference values of Todt et al. (1996).

\section{Results}

Present-day Hf-Nd isotopic values are plotted in Fig. 3 together with the global mantle array $(\varepsilon \mathrm{Hf}=1.4 \varepsilon$ $\mathrm{Nd}+2.8$; Blichert-Toft, unpublished compilation). The ${ }^{176} \mathrm{Hf} /{ }^{177} \mathrm{Hf}$ isotopic ratios span a large range (over $14 \varepsilon \mathrm{Hf}$ units in contrast to only $6 \varepsilon N d$ units). Samples from the Madeira archipelago are most radiogenic, largely overlapping the field for central Atlantic MORB. They seem to form a relatively narrow, elongated trend (stretching over $>6$ \&Hf units) between a composition similar to the radiogenic MORB endmember and a composition located on or slightly below the mantle array. Samples from Seine and Unicorn seamounts, attributed to the Madeira hotspot track or "Madeira domain", show less radiogenic Hf isotope ratios than the Madeira archipelago samples. Their isotopic compositions, however, lie along an extension of the Madeira trend. This observation generally holds also for plots of $\mathrm{Hf}$ versus $\mathrm{Pb}$ and $\mathrm{Sr}$ isotope compositions (Fig. 4). In contrast to Madeira, all Canary archipelago samples plot below the mantle array, despite representing many islands from a larger geographic area, and form a dense cluster with less compositional variation $(\sim 4 \varepsilon \mathrm{Hf}$ units). All samples from the seamounts northeast of the Canary Islands, proposed to belong to the same Canary hotspot track or "Canary domain", fall within the same ${ }^{176} \mathrm{Hf} /{ }^{177} \mathrm{Hf}$ isotopic range of this cluster (with no apparent geographic-compositional relationship). Interestingly, the cluster partly overlaps the proposed common mantle endmember "C" composition (Hanan and Graham 1996) and seems to span a space between " $\mathrm{C}$ " and a more radiogenic, depleted composition (having higher $\mathrm{Hf}$ and $\mathrm{Nd}$ isotope ratios) and a HIMU-like endmember (having low ${ }^{176} \mathrm{Hf} /{ }^{177} \mathrm{Hf}$ for a given ${ }^{143} \mathrm{Nd} /{ }^{144} \mathrm{Nd}$ and falling well below the mantle array). Note that the two samples from the Cretaceous Madeira-Tore Rise basement (Lion and Teresa) plot well within the Canary cluster. This compositional 
Table 1 Sample locations and $\mathrm{Hf}$ isotope ratios

\begin{tabular}{|c|c|c|c|c|c|c|c|c|}
\hline Sample & Location & Coordinates & Volcanic stage & Age (Ma) & Ref. & Anal. & ${ }^{176} \mathrm{Hf} /{ }^{177} \mathrm{Hf}$ & $\varepsilon \mathrm{Hf}$ \\
\hline DS797-1B & Ampere Smt. & $35^{\circ} 03^{\prime} 12^{\prime \prime} \mathrm{N} ; 12^{\circ} 54^{\prime} 00^{\prime \prime} \mathrm{W}$ & Shield St. & 31.2 & 1,2 & $\mathrm{~L}$ & 0.282998 & 8.0 \\
\hline VH-97-103 & Ampere Smt. & $35^{\circ} 03^{\prime} 01^{\prime \prime} \mathrm{N} ; 12^{\circ} 54^{\prime} 02^{\prime \prime} \mathrm{W}$ & Shield St. & $31 ?$ & 1,2 & $\mathrm{~L}$ & $0.282999(7)$ & 8.0 \\
\hline 423 DR-1 & Unicorn Smt. & $34^{\circ} 47^{\prime} 25^{\prime \prime} \mathrm{N} ; 14^{\circ} 35^{\prime} 17^{\prime \prime} \mathrm{W}$ & Shield St. & 24.75 & 4 & $\mathrm{~K}$ & $0.283080(8)$ & 10.9 \\
\hline 426 DR-1 & Seine Smt. & $33^{\circ} 52^{\prime} 04^{\prime \prime} \mathrm{N} ; 14^{\circ} 22^{\prime} 08^{\prime \prime} \mathrm{W}$ & Shield St. & 21.77 & 4 & $\mathrm{~K}$ & $0.283034(5)$ & 9.3 \\
\hline 426 DR-2 & Seine Smt. & $33^{\circ} 52^{\prime} 04^{\prime \prime} \mathrm{N} ; 14^{\circ} 22^{\prime} 08^{\prime \prime} \mathrm{W}$ & Shield St. & $21 ?$ & 4 & $\mathrm{~K}$ & $0.283030(6)$ & 9.1 \\
\hline K 43 & Porto Santo & $33^{\circ} 05^{\prime} 23^{\prime \prime} \mathrm{N} ; 16^{\circ} 19^{\prime} 09^{\prime \prime} \mathrm{W}$ & Shield St.? & 11.1 & 1,2 & $\mathrm{~L}$ & $0.283239(7)$ & 16.5 \\
\hline Ma 85 & Madeira & $32^{\circ} 49^{\prime} 08^{\prime \prime} \mathrm{N} ; 17^{\circ} 02^{\prime} 28^{\prime \prime} \mathrm{W}$ & Late St. & 0.2 & 1,2 & $\mathrm{~L}$ & $0.283335(7)$ & 19.9 \\
\hline Ma 88 & Madeira & $32^{\circ} 49^{\prime} 00^{\prime \prime} \mathrm{N} ; 17^{\circ} 06^{\prime} 23^{\prime \prime} \mathrm{W}$ & Late St. & $0.2 ?$ & 1,2 & $\mathrm{~L}$ & $0.283321(7)$ & 19.4 \\
\hline Ma 88 (Repl. 1) & & & & & & $\mathrm{K}$ & $0.283321(8)$ & 19.4 \\
\hline Ma 88 (Repl. 2) & & & & & & $\mathrm{K}$ & $0.283320(6)$ & 19.4 \\
\hline Ma 218a & Madeira & $32^{\circ} 45^{\prime} 05^{\prime \prime} \mathrm{N} ; 17^{\circ} 03^{\prime} 10^{\prime \prime} \mathrm{W}$ & Late St. & 0 & 1,2 & $\mathrm{~L}$ & $0.283301(6)$ & 18.7 \\
\hline 13796-1 & Madeira & $32^{\circ} 44^{\prime} 53^{\prime \prime} \mathrm{N} ; 16^{\circ} 48^{\prime} 42^{\prime \prime} \mathrm{W}$ & Shield St. & 4.5 & 1,2 & $\mathrm{~L}$ & $0.283170(12)$ & 14.1 \\
\hline Ma 227 & Madeira & $32^{\circ} 43^{\prime} 58^{\prime \prime} \mathrm{N} ; 16^{\circ} 57^{\prime} 53^{\prime \prime} \mathrm{W}$ & Shield St. & 3.9 & 1,2 & $\mathrm{~L}$ & $0.283227(8)$ & 16.1 \\
\hline Ma 215 & Madeira & $32^{\circ} 43^{\prime} 26^{\prime \prime} \mathrm{N} ; 17^{\circ} 01^{\prime} 43^{\prime \prime} \mathrm{W}$ & Shield St. & $4.5 ?$ & 1,2 & $\mathrm{~L}$ & $0.283168(7)$ & 14.0 \\
\hline Ma 17 & Madeira & $32^{\circ} 44^{\prime} 50^{\prime \prime} \mathrm{N} ; 17^{\circ} 01^{\prime} 33^{\prime \prime} \mathrm{W}$ & Shield St. & $1 ?$ & 1,2 & $\mathrm{~L}$ & $0.283223(7)$ & 16.0 \\
\hline Ma 17 (Repl. 1) & & & & & & $\mathrm{K}$ & $0.283222(7)$ & 15.9 \\
\hline Ma 17 (Repl. 2) & & & & & & $\mathrm{K}$ & $0.283234(4)$ & 16.3 \\
\hline Ma 23 & Madeira & $32^{\circ} 44^{\prime} 38^{\prime \prime} \mathrm{N} ; 17^{\circ} 01^{\prime} 51^{\prime \prime} \mathrm{W}$ & Shield St. & $1 ?$ & 1,2 & $\mathrm{~L}$ & $0.283207(7)$ & 15.4 \\
\hline Ma 75b & Madeira & $32^{\circ} 45^{\prime} 06^{\prime \prime} \mathrm{N} ; 17^{\circ} 01^{\prime} 07^{\prime \prime} \mathrm{W}$ & Shield St. & 1.8 & 1,2 & $\mathrm{~L}$ & $0.283205(8)$ & 15.3 \\
\hline Ma 160 & Madeira & $32^{\circ} 43^{\prime} 46^{\prime \prime} \mathrm{N} ; 17^{\circ} 11^{\prime} 43^{\prime \prime} \mathrm{W}$ & Shield St. & $1.5 ?$ & 1,2 & $\mathrm{~L}$ & $0.283308(8)$ & 19.0 \\
\hline DGR 47 & Deserta Grande & $32^{\circ} 32^{\prime} 23^{\prime \prime} \mathrm{N} ; 16^{\circ} 31^{\prime} 21^{\prime \prime} \mathrm{W}$ & Shield St. & 3.4 & 1,2 & $\mathrm{~L}$ & $0.283132(6)$ & 12.7 \\
\hline K 11 & Deserta Bugio & $32^{\circ} 25^{\prime} 56^{\prime \prime} \mathrm{N} ; 16^{\circ} 29^{\prime} 17^{\prime \prime} \mathrm{W}$ & Shield St. & 3.4 & 1,2 & $\mathrm{~L}$ & $0.283153(8)$ & 13.5 \\
\hline DS $832-2$ & Lars Smt. & $32^{\circ} 48^{\prime} 05^{\prime \prime} \mathrm{N} ; 13^{\circ} 17^{\prime} 10^{\prime \prime} \mathrm{W}$ & Late St. & 67.7 & 3 & SD & $0.282942(2)$ & 6.0 \\
\hline DS $809-1$ & Dacia Smt. & $31^{\circ} 08^{\prime} 03^{\prime \prime} \mathrm{N} ; 13^{\circ} 31^{\prime} 06^{\prime \prime} \mathrm{W}$ & Late St. & 9.2 & 3 & SD & $0.283031(2)$ & 9.2 \\
\hline DS $830-2 a$ & Nico Smt. & $30^{\circ} 26^{\prime} 26^{\prime \prime} \mathrm{N} ; 13^{\circ} 20^{\prime} 33^{\prime \prime} \mathrm{W}$ & Late St. & $20 ?$ & 3 & SD & 0.283031 & 9.2 \\
\hline DS $836-2$ & Last Minute Smt. & $30^{\circ} 09^{\prime} 32^{\prime \prime} \mathrm{N} ; 14^{\circ} 44^{\prime} 35^{\prime \prime} \mathrm{W}$ & Shield St. & $30 ?$ & 3 & $\mathrm{SD}$ & 0.283000 & 8.1 \\
\hline SG 10 & Selvagen Grande & $30^{\circ} 08^{\prime} 40^{\prime \prime} \mathrm{N} ; 15^{\circ} 52^{\prime} 15^{\prime \prime} \mathrm{W}$ & Late St. & 3.4 & 3 & SD & $0.283029(2)$ & 9.1 \\
\hline J 30 & Selvagen Grande & $30^{\circ} 08^{\prime} 13^{\prime \prime} \mathrm{N} ; 15^{\circ} 51^{\prime} 43^{\prime \prime} \mathrm{W}$ & Late St. & 3.4 & 3 & SD & $0.283027(2)$ & 9.0 \\
\hline $\mathrm{J} 35 \mathrm{~g}$ & Selvagen Grande & $30^{\circ} 08^{\prime} 19^{\prime \prime} \mathrm{N} ; 15^{\circ} 51^{\prime} 56^{\prime \prime} \mathrm{W}$ & Shield St. & 24.8 & 3 & $\mathrm{SD}$ & $0.283043(2)$ & 9.6 \\
\hline $\mathrm{J} 41 \mathrm{a}$ & Selvagen Grande & $30^{\circ} 08^{\prime} 22^{\prime \prime} \mathrm{N} ; 15^{\circ} 51^{\prime} 30^{\prime \prime} \mathrm{W}$ & Shield St. & 25.8 & 3 & $\mathrm{SD}$ & $0.283044(2)$ & 9.6 \\
\hline J 56 & Selvagen Pequ. & $30^{\circ} 18^{\prime} 00^{\prime \prime} \mathrm{N} ; 16^{\circ} 10^{\prime} 05^{\prime \prime} \mathrm{W}$ & Shield St. & 29 & 3 & $\mathrm{SD}$ & $0.283033(2)$ & 9.2 \\
\hline DS $822-4$ & Conception Smt. & $29^{\circ} 49^{\prime} 21^{\prime \prime} \mathrm{N} ; 12^{\circ} 39^{\prime} 42^{\prime \prime} \mathrm{W}$ & Late St. & 16.6 & 3 & $\mathrm{SD}$ & $0.283041(2)$ & 9.5 \\
\hline DS $822-9$ & Conception Smt. & $29^{\circ} 49^{\prime} 21^{\prime \prime} \mathrm{N} ; 12^{\circ} 39^{\prime} 42^{\prime \prime} \mathrm{W}$ & Late St. & $17 ?$ & 3 & $\mathrm{SD}$ & $0.283049(1)$ & 9.8 \\
\hline EL 3 & Lanzarote & $29^{\circ} 01^{\prime} 07^{\prime \prime} \mathrm{N} ; 13^{\circ} 43^{\prime} 05^{\prime \prime} \mathrm{W}$ & Late St. & $<0.1$ & 5 & SD & $0.283019(2)$ & 8.7 \\
\hline EL 11 & Lanzarote & $28^{\circ} 59^{\prime} 18^{\prime \prime} \mathrm{N} ; 13^{\circ} 41^{\prime} 22^{\prime \prime} \mathrm{W}$ & Late St. & $<0.1$ & 5 & $\mathrm{SD}$ & $0.283038(2)$ & 9.4 \\
\hline EL 13 & Lanzarote & $28^{\circ} 57^{\prime} 13^{\prime \prime} \mathrm{N} ; 13^{\circ} 46^{\prime} 57^{\prime \prime} \mathrm{W}$ & Late St. & $<0.1$ & 5 & SD & $0.283015(2)$ & 8.6 \\
\hline EL 1 & Lanzarote & $29^{\circ} 02^{\prime} 54^{\prime \prime} \mathrm{N} ; 13^{\circ} 37^{\prime} 04^{\prime \prime} \mathrm{W}$ & Late St. & $<0.1$ & 5 & SD & $0.283034(3)$ & 9.3 \\
\hline EL 5 & Lanzarote & $29^{\circ} 01^{\prime} 30^{\prime \prime} \mathrm{N} ; 13^{\circ} 44^{\prime} 17^{\prime \prime} \mathrm{W}$ & Late St. & $<0.1$ & 5 & $\mathrm{SD}$ & $0.283012(3)$ & 8.5 \\
\hline EL 8 & Lanzarote & $29^{\circ} 00^{\prime} 28^{\prime \prime} \mathrm{N} ; 13^{\circ} 40^{\prime} 46^{\prime \prime} \mathrm{W}$ & Late St. & $<0.1$ & 5 & SD & $0.283028(2)$ & 9.1 \\
\hline EL 10 & Lanzarote & $28^{\circ} 59^{\prime} 27^{\prime \prime} \mathrm{N} ; 13^{\circ} 41^{\prime} 23^{\prime \prime} \mathrm{W}$ & Late St. & $<0.1$ & 5 & SD & $0.283058(2)$ & 10.1 \\
\hline EL 17 & Lanzarote & $29^{\circ} 10^{\prime} 53^{\prime \prime} \mathrm{N} ; 13^{\circ} 27^{\prime} 00^{\prime \prime} \mathrm{W}$ & Late St. & $<0.1$ & 5 & SD & $0.282987(2)$ & 7.6 \\
\hline ELP 1 & La Palma & $28^{\circ} 38^{\prime} 32^{\prime \prime} \mathrm{N} ; 14^{\circ} 09^{\prime} 18^{\prime \prime} \mathrm{W}$ & Shield St. & $<0.1$ & 5 & SD & $0.282943(2)$ & 6.0 \\
\hline LP 71-7L & La Palma & & Shield St. & $<0.1$ & 5 & SD & $0.282953(3)$ & 6.4 \\
\hline ELP 4 & La Palma & $28^{\circ} 45^{\prime} 40^{\prime \prime} \mathrm{N} ; 14^{\circ} 16^{\prime} 21^{\prime \prime} \mathrm{W}$ & Shield St. & $<0.1$ & 5 & SD & $0.282960(2)$ & 6.6 \\
\hline ELP 7 & La Palma & $28^{\circ} 31^{\prime} 03^{\prime \prime} \mathrm{N} ; 14^{\circ} 09^{\prime} 56^{\prime \prime} \mathrm{W}$ & Shield St. & $<0.1$ & 5 & SD & $0.282964(2)$ & 6.8 \\
\hline LP 124794 & La Palma & & Shield St. & $<0.1$ & 5 & SD & $0.282960(2)$ & 6.7 \\
\hline LP 261925 & La Palma & & Shield St. & $<0.1$ & 5 & SD & $0.282966(2)$ & 6.9 \\
\hline EF 1 & Fuerteventura & $28^{\circ} 19^{\prime} 36^{\prime \prime} \mathrm{N} ; 13^{\circ} 54^{\prime} 29^{\prime \prime} \mathrm{W}$ & Shield St. & $<0.1$ & 5 & SD & $0.283067(5)$ & 10.4 \\
\hline
\end{tabular}


Table 1 continued

\begin{tabular}{|c|c|c|c|c|c|c|c|c|}
\hline Sample & Location & Coordinates & Volcanic stage & Age (Ma) & Ref. & Anal. & ${ }^{176} \mathrm{Hf} /{ }^{177} \mathrm{Hf}$ & $\varepsilon \mathrm{Hf}$ \\
\hline EF 5 & Fuerteventura & $28^{\circ} 21^{\prime} 23^{\prime \prime} \mathrm{N} ; 14^{\circ} 00^{\prime} 44^{\prime \prime} \mathrm{W}$ & Shield St. & $<0.1$ & 5 & $\mathrm{SD}$ & $0.283046(3)$ & 9.7 \\
\hline EF 6 & Fuerteventura & $28^{\circ} 35^{\prime} 15^{\prime \prime} \mathrm{N} ; 13^{\circ} 49^{\prime} 59^{\prime \prime} \mathrm{W}$ & Late St. & $<0.1$ & 5 & $\mathrm{SD}$ & $0.283019(2)$ & 8.8 \\
\hline EF 7 & Fuerteventura & $28^{\circ} 43^{\prime} 16^{\prime \prime} \mathrm{N} ; 13^{\circ} 52^{\prime} 52^{\prime \prime} \mathrm{W}$ & Late St. & $<0.1$ & 5 & $\mathrm{SD}$ & $0.283043(2)$ & 9.6 \\
\hline EF 12 & Fuerteventura & $28^{\circ} 43^{\prime} 37^{\prime \prime} \mathrm{N} ; 13^{\circ} 58^{\prime} 40^{\prime \prime} \mathrm{W}$ & Late St. & $<0.1$ & 5 & $\mathrm{SD}$ & 0.283011 & 8.4 \\
\hline Tf 1577 & Tenerife & & Late St. & $<0.1$ & 5 & $\mathrm{SD}$ & 0.282963 & 6.7 \\
\hline ET 2 & Tenerife & $28^{\circ} 26^{\prime} 37^{\prime \prime} \mathrm{N} ; 12^{\circ} 46^{\prime} 26^{\prime \prime} \mathrm{W}$ & Late St. & $<0.1$ & 5 & $\mathrm{SD}$ & $0.282964(2)$ & 6.8 \\
\hline ET 6 & Tenerife & $28^{\circ} 19^{\prime} 37^{\prime \prime} \mathrm{N} ; 12^{\circ} 45^{\prime} 10^{\prime \prime} \mathrm{W}$ & Late St. & $<0.1$ & 5 & SD & 0.282963 & 6.7 \\
\hline ET 8 & Tenerife & $28^{\circ} 17^{\prime} 20^{\prime \prime} \mathrm{N} ; 12^{\circ} 40^{\prime} 32^{\prime \prime} \mathrm{W}$ & Late St. & $<0.1$ & 5 & $\mathrm{SD}$ & $0.282966(2)$ & 6.9 \\
\hline EGC 1 & Gran Canaria & $27^{\circ} 56^{\prime} 44^{\prime \prime} \mathrm{N} ; 15^{\circ} 26^{\prime} 36^{\prime \prime} \mathrm{W}$ & Late St. & $<0.1$ & 5 & $\mathrm{SD}$ & 0.282983 & 7.5 \\
\hline EGC 3 & Gran Canaria & $28^{\circ} 01^{\prime} 34^{\prime \prime} \mathrm{N} ; 15^{\circ} 26^{\prime} 00^{\prime \prime} \mathrm{W}$ & Late St. & $<0.1$ & 5 & $\mathrm{SD}$ & $0.282997(8)$ & 8.0 \\
\hline EGC 6 & Gran Canaria & $28^{\circ} 01^{\prime} 52^{\prime \prime} \mathrm{N} ; 15^{\circ} 35^{\prime} 57^{\prime \prime} \mathrm{W}$ & Late St. & $<0.1$ & 5 & $\mathrm{SD}$ & $0.282982(3)$ & 7.4 \\
\hline EGC 8 & Gran Canaria & $28^{\circ} 57^{\prime} 10^{\prime \prime} \mathrm{N} ; 15^{\circ} 31^{\prime} 26^{\prime \prime} \mathrm{W}$ & Late St. & $<0.1$ & 5 & $\mathrm{SD}$ & 0.282994 & 7.9 \\
\hline GCQ 1369 & Gran Canaria & & Late St. & $<0.1$ & 5 & $\mathrm{SD}$ & 0.282988 & 7.7 \\
\hline EH 9 & Hierro & $27^{\circ} 39^{\prime} 48^{\prime \prime} \mathrm{N} ; 18^{\circ} 01^{\prime} 00^{\prime \prime} \mathrm{W}$ & Shield St. & $<0.1$ & 5 & $\mathrm{SD}$ & 0.283018 & 8.7 \\
\hline EH 11 & Hierro & $27^{\circ} 39^{\prime} 24^{\prime \prime} \mathrm{N} ; 17^{\circ} 59^{\prime} 49^{\prime \prime} \mathrm{W}$ & Shield St. & $<0.1$ & 5 & $\mathrm{SD}$ & $0.283007(2)$ & 8.3 \\
\hline EH 4 & Hierro & $27^{\circ} 45^{\prime} 46^{\prime \prime} \mathrm{N} ; 17^{\circ} 54^{\prime} 50^{\prime \prime} \mathrm{W}$ & Shield St. & $<0.1$ & 5 & SD & $0.282983(2)$ & 7.5 \\
\hline EH 4 Duplikat & Hierro & & Shield St. & $<0.1$ & 5 & SD & $0.282987(3)$ & 7.6 \\
\hline EH 8 & Hierro & $27^{\circ} 45^{\prime} 16^{\prime \prime} \mathrm{N} ; 18^{\circ} 08^{\prime} 54^{\prime \prime} \mathrm{W}$ & Shield St. & $<0.1$ & 5 & $\mathrm{SD}$ & 0.282995 & 7.9 \\
\hline
\end{tabular}

Question mark signalizes that the given ages are not based on direct radiometric age determinations but rather on field relationships and are therefore uncertain. Reference for sample location, age and respective Nd, Pb and Sr isotope data: (1) Geldmacher et al. (2000); (2) Geldmacher and Hoernle (2000); (3) Geldmacher et al. (2001); (4) Geldmacher et al. (2005); (5) this study (see Table 2). Anal. = Hf isotope data measured in Lyon (L), Kiel (K) or San Diego (SD). eHf is calculated with ${ }^{176} \mathrm{Hf} /{ }^{177} \mathrm{Hf}_{\mathrm{CHUR}}=0.282772$ (Blichert-Toft and Albarède 1997)

relationship and the general contrast between the two Madeira and Canary domains are also seen for plots of Hf isotopes versus all other available isotope systems (Fig. 4).

\section{Discussion}

Genetic relationships

The new Hf isotope data show that East Atlantic intraplate volcanism samples can be divided into two separate groups in any radiogenic isotope plot, emphasizing the proposed spatial-compositionally distinct Madeira and Canary domains. Noticeable exceptions are samples from the NE part of the proposed Madeira hotspot trace, which are less distinct (Unicorn, Seine) or even overlap (Ampere, Ormonde) with the Canary domain Hf isotope composition (Figs. 3, and 4). As mentioned above, one possible explanation could be a progressive influence of continental lithosphere to the Madeira hotspot source with decreasing distance to the Eurasian continental shelf (Geldmacher et al. 2000). Although such a process is obvious for proximal Ormonde Seamount (Fig. 1), the underlying mechanism is less clear for Seine and Unicorn located $>400 \mathrm{~km}$ from the nearest continental shelf (Fig. 1). Alternatively, the early path of the Madeira hotspot was influenced by material from the nearby Canary hotspot. The Madeira-Tore Rise may have formed as an aseismic ridge by sublithospheric flow of Canary plume material at a time when the migrating MidAtlantic spreading center was above or near the Canary hotspot (Geldmacher et al. 2006a). This model is supported by the two Cretaceous samples from the Cretaceous Madeira-Tore Rise basement (Lion and Teresa) plotting well within the Canary domain cluster in all isotope diagrams (Figs. 2, 3, and 4). Eventually, the flow of plume material to the departing ridge ceased and a new hotspot track formed (at some distance from the ridge) on the new, overriding plate (Sleep 2002). The path of the Madeira hotspot crossed the gap between the Madeira-Tore Rise and the proposed onset of the Canary hotspot track (at Essaouira seamount) on the African plate (Holik et al. 1991; Geldmacher et al. 2001, 2005). Rising magmas from the Madeira hotspot could have mobilized and mingled with residual Canary plume material that was frozen at the base of the lithosphere. The Madeira domain magma source may have developed its compositional distinctness with increasing distance from the described Madeira-Tore Rise-Canary domain corridor.

\section{Origin and interaction of the Madeira and Canary} magma sources

It is generally accepted that isotopic variations in mantle sources of intraplate volcanic rocks are largely caused by recycling of lithospheric material (e.g. Armstrong 1968; 
Table 2 Corresponding $\mathrm{Sr}, \mathrm{Nd}$, and $\mathrm{Pb}$ isotope ratios of Canary Islands samples

\begin{tabular}{|c|c|c|c|c|c|c|}
\hline Sample & & ${ }^{87} \mathrm{Sr} /{ }^{86} \mathrm{Sr}$ & ${ }^{143} \mathrm{Nd} /{ }^{144} \mathrm{Nd}$ & ${ }^{206} \mathrm{~Pb} /{ }^{204} \mathrm{~Pb}$ & ${ }^{207} \mathrm{~Pb} /{ }^{204} \mathrm{~Pb}$ & ${ }^{208} \mathrm{~Pb} /{ }^{204} \mathrm{~Pb}$ \\
\hline EL1 & Lanzarote & $0.703258(10)$ & $0.512895(10)$ & $19.340(2)$ & $15.576(2)$ & 39.178 (4) \\
\hline EL3 & Lanzarote & $0.703154(10)$ & $0.512929(7)$ & $19.163(1)$ & $15.564(1)$ & $38.999(2)$ \\
\hline EL5 & Lanzarote & $0.703050(6)$ & $0.512920(7)$ & $19.188(1)$ & $15.558(1)$ & $38.974(3)$ \\
\hline EL8* & Lanzarote & 0.703052 & 0.512921 & $19.225(2)$ & $15.556(2)$ & $39.050(5)$ \\
\hline EL10 & Lanzarote & $0.703040(10)$ & $0.512996(7)$ & $19.227(1)$ & $15.549(1)$ & $38.964(4)$ \\
\hline EL11 & Lanzarote & 0.703018 (9) & $0.512987(8)$ & $19.224(1)$ & $15.552(1)$ & $38.991(5)$ \\
\hline EL13 & Lanzarote & $0.703136(8)$ & $0.512946(7)$ & $19.162(1)$ & $15.562(1)$ & $39.017(2)$ \\
\hline EL17 & Lanzarote & $0.703236(11)$ & $0.512886(7)$ & $19.353(1)$ & $15.575(1)$ & $39.182(5)$ \\
\hline ELP4* & La Palma & 0.703057 & 0.512911 & $19.487(1)$ & $15.573(1)$ & $39.188(2)$ \\
\hline ELP7* & La Palma & 0.703110 & $0.512896(6)$ & $19.660(1)$ & $15.588(1)$ & $39.437(2)$ \\
\hline LP124794 & La Palma & 0.703097 (11) & $0.512903(9)$ & $19.618(11)$ & $15.604(9)$ & $39.413(22)$ \\
\hline LP261925 & La Palma & $0.703121(14)$ & 0.512911 & $19.673(1)$ & $15.620(1)$ & $39.515(4)$ \\
\hline ELP1 & La Palma & $0.703078(8)$ & $0.512897(8)$ & $19.635(2)$ & $15.596(1)$ & $39.435(5)$ \\
\hline LP71-7L & La Palma & $0.703118(8)$ & $0.512922(7)$ & $19.611(3)$ & $15.600(2)$ & $39.392(2)$ \\
\hline $\mathrm{EF} 1$ & Fuerteventura & $0.702982(8)$ & $0.513022(8)$ & $19.098(3)$ & $15.549(3)$ & $38.861(7)$ \\
\hline EF5* & Fuerteventura & 0.703028 & 0.512961 & $19.201(1)$ & $15.541(1)$ & $38.861(2)$ \\
\hline EF6 & Fuerteventura & $0.703188(10)$ & $0.512904(7)$ & $19.194(3)$ & $15.559(3)$ & $38.997(7)$ \\
\hline EF7 & Fuerteventura & 0.703052 (17) & $0.512956(18)$ & $19.160(2)$ & $15.541(2)$ & $38.944(4)$ \\
\hline EF12 & Fuerteventura & $0.703187(11)$ & $0.512884(7)$ & $19.202(1)$ & $15.573(1)$ & 39.108 (3) \\
\hline ET2 & Tenerife & $0.703038(11)$ & $0.512901(8)$ & $19.718(2)$ & $15.597(2)$ & $39.490(4)$ \\
\hline ET6 & Tenerife & $0.703103(8)$ & $0.512907(7)$ & $19.713(1)$ & $15.599(1)$ & $39.476(4)$ \\
\hline ET8 & Tenerife & $0.703149(11)$ & $0.512885(9)$ & $19.739(2)$ & $15.599(1)$ & $39.491(3)$ \\
\hline Tf1577 & Tenerife & $0.703066(11)$ & $0.512903(7)$ & $19.730(3)$ & $15.615(2)$ & 39.538 (7) \\
\hline EGC1* & Gran Canaria & 0.703209 (3) & 0.512878 & $19.481(1)$ & $15.584(1)$ & $39.340(2)$ \\
\hline EGC3 & Gran Canaria & $0.703161(32)$ & $0.512923(8)$ & $19.594(4)$ & $15.579(4)$ & $39.382(10)$ \\
\hline EGC6 & Gran Canaria & $0.703172(14)$ & 0.512938 & $19.556(1)$ & $15.579(1)$ & $39.383(3)$ \\
\hline EGC8* & Gran Canaria & 0.703153 & $0.512900(7)$ & $19.380(1)$ & $15.571(1)$ & $39.208(2)$ \\
\hline GCQ1369* & Gran Canaria & 0.703147 & 0.512887 & $19.358(1)$ & $15.575(1)$ & $39.181(3)$ \\
\hline EH4 & Hierro & $0.703019(11)$ & $0.512970(6)$ & $19.413(2)$ & $15.564(2)$ & 39.059 (4) \\
\hline EH8 & Hierro & $0.702924(13)$ & $0.512974(8)$ & $19.396(1)$ & $15.589(1)$ & 39.039 (4) \\
\hline EH9* & Hierro & 0.702958 & 0.512968 & $19.180(1)$ & $15.553(1)$ & $38.798(2)$ \\
\hline EH11 & Hierro & $0.702961(11)$ & $0.512940(9)$ & $19.355(3)$ & $15.560(3)$ & $39.004(7)$ \\
\hline
\end{tabular}

* Indicate samples measured at IFM-GEOMAR Kiel. All other samples were run at UCSC. See Table 1 for sample location and age information

Zindler and Hart 1986; Hofmann 2003; Sobolev et al. 2007) with the classical HIMU endmember being attributed to recycling of the basaltic portion of subducted oceanic crust (e.g. Hofmann and White 1982; Zindler et al. 1982; Chauvel et al. 1992). MORB basalts have a lower $\mathrm{Lu} / \mathrm{Hf}$ ratio than their source (because part of the melting takes place in the presence of garnet that retains HREEs; Salters and Hart 1989; Hirschmann and Stolper 1996), leading to a time-integrated decoupling of $\mathrm{Hf}$ from $\mathrm{Nd}$ isotope ratios (e.g. Salters and White 1998). The older the melting event that formed the crust, the more the ${ }^{176} \mathrm{Hf} /{ }^{177} \mathrm{Hf}$ ratio lags behind, resulting in isotopic compositions that plot progressively farther below the Nd-Hf mantle array (black squares in Fig. 5). Therefore, the different $\mathrm{Hf}$ isotope ratios of the respective enriched endmembers for the Madeira and Canary domains could be explained simply by a different age of the recycled crust component in their specific magma sources. It should be noted, however, that subducted crust also comprises sediments that may be stored in varying proportions together with the basaltic protolith. Although subducted (pelagic) sediment has a composition above and to the far left of the mantle array (i.e., radiogenic $\mathrm{Hf}$ for a given $\mathrm{Nd}$ isotopic composition; $\varepsilon \mathrm{Hf} \approx+2, \varepsilon \mathrm{Nd} \approx-8.9$, Plank and Langmuir 1998; Vervoort et al. 1999; Chauvel et al. 2008), mixtures of recycled crust containing less then 10-15\% sediment will still plot below the mantle array (Stracke et al. 2003; Fig. 5, this study). Chauvel et al. (2008) proposed that the $\mathrm{Nd}$ and $\mathrm{Hf}$ isotope characteristics of all ocean islands forming the mantle array can be explained by 


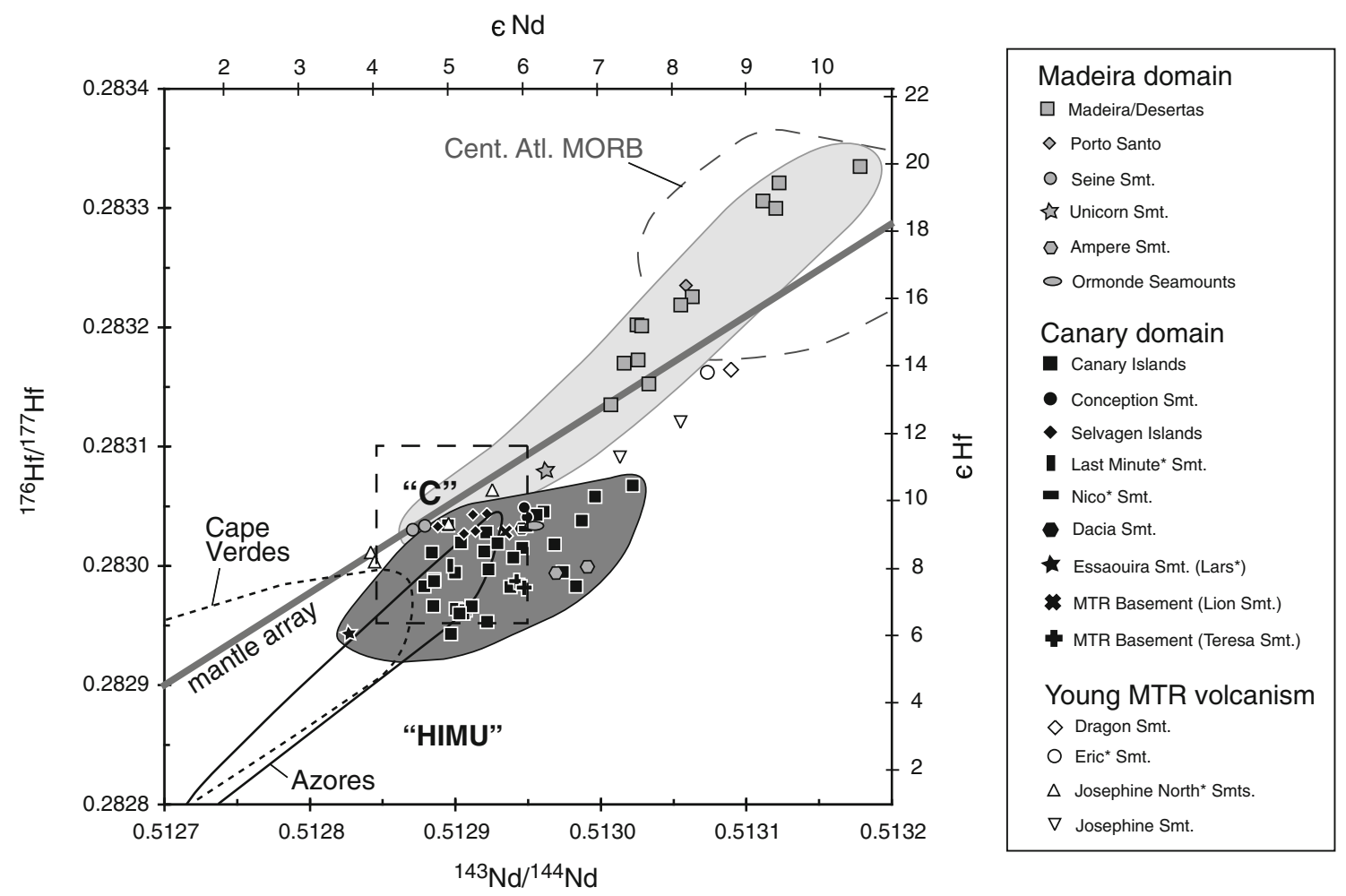

Fig. $3{ }^{143} \mathrm{Nd} /{ }^{144} \mathrm{Nd}$ versus ${ }^{176} \mathrm{Hf} /{ }^{177} \mathrm{Hf}$ systematics for Madeira and Canary domain samples (Madeira-Tore Rise data from Geldmacher et al. (2006a)). Nd/Hf OIB mantle array based on $\varepsilon \mathrm{Hf}=1.4 \varepsilon \mathrm{Nd}+2.8 \quad$ (J. Blichert-Toft, unpublished compilation).

mixtures of recycled oceanic basalt with varying proportions of similarly aged subducted sediments, subsequently mixed with depleted mantle peridotite. Following this simple model, the enriched, HIMU-like endmember in the Canary domain could be explained by mixtures of slightly $>1$-Ga-old recycled oceanic crust with minor but variable additions (0-10\%) of similarly old sediment (Fig. 5). This would be consistent with combined $\mathrm{Pb}$ and $\mathrm{Os}$ isotope studies suggesting an age of $1.2 \mathrm{Ga}$ for the enriched Canary Island endmember (Widom et al. 1999). The narrow Madeira domain field would require a more restricted proportion (6-8\%) of sediment addition for its enriched endmember. It is unclear to which extent the recycled material is reflected in the most enriched available samples, but a recycling age of $<1 \mathrm{Ga}$ for the subducted crust/sediment component seems reasonable (Fig. 5), consistent with a proposed recycling age of $<1 \mathrm{Ga}$ inferred from $\mathrm{Nd}$ and $\mathrm{Pb}$ isotope ratios of Madeira samples that plot well below the NHRL (Geldmacher and Hoernle 2000).

It remains a matter of debate to what extent any subducted sediment can contribute to a HIMU-like source without significantly impacting its $\mathrm{Pb}$ and $\mathrm{Sr}$ isotopic compositions (see discussion in Stracke et al. 2003). Because of the highly different abundances of the trace elements $\mathrm{Sr}$ and $\mathrm{Pb}$ in sediment versus MORB, any
Common mantle endmember "C" composition after Hanan and Graham (1996) with ${ }^{176} \mathrm{Hf} /{ }^{177} \mathrm{Hf}$ ratios $(0.28295-0.28310)$ based on Hanan et al. (2000). See Fig. 2 for symbols, fields, and additional Nd data sources

sediment contribution that is sufficient to move subducted ocean crust compositions toward the OIB array on $\mathrm{Nd}$ vs Hf isotope plots (Fig. 5) should also result in a noticeable trend toward low ${ }^{206} \mathrm{~Pb} /{ }^{204} \mathrm{~Pb}$ ratios and high ${ }^{86} \mathrm{Sr} /{ }^{87} \mathrm{Sr}$ ratios (e.g. Stracke et al. 2003), which is not observed in Fig. 4b, c. On the other hand, the isotopic effect of sediment addition depends on the process responsible for imparting the sediment signature (e.g. bulk sediment contribution, hydrothermal exchange, or sea water alteration). One explanation could be the postulated decoupling of the $\mathrm{Lu}-\mathrm{Hf}$ from the $\mathrm{Rb}-\mathrm{Sr}$ and $\mathrm{U}-\mathrm{Pb}$ isotope systems during the subduction process due to preferential removal of $\mathrm{Pb}$ and $\mathrm{Rb}$ relative to $\mathrm{U}$ and $\mathrm{Sr}$ (e.g. Kelley et al. 2005). A further explanation could be related to the assumed young age of the recycled sedimentary material involved $(<1.2 \mathrm{Ga})$.

The lower $\mathrm{Hf}$ and $\mathrm{Nd}$ but higher $\mathrm{Pb}$ isotope ratios of the Canary domain samples (compared to Madeira) cannot just reflect an increased contribution of the enriched recycled component in the magma source. It is suggested that such enriched components exist as pyroxenitic or eclogitic veins (gt-pyroxenites) within the depleted peridotitic mantle matrix (e.g. Zindler et al. 1984; Allègre and Turcotte 1986; Hirschmann and Stolper 1996). To assess the contribution of peridotitic versus recycled pyroxenitic-eclogitic mantle 

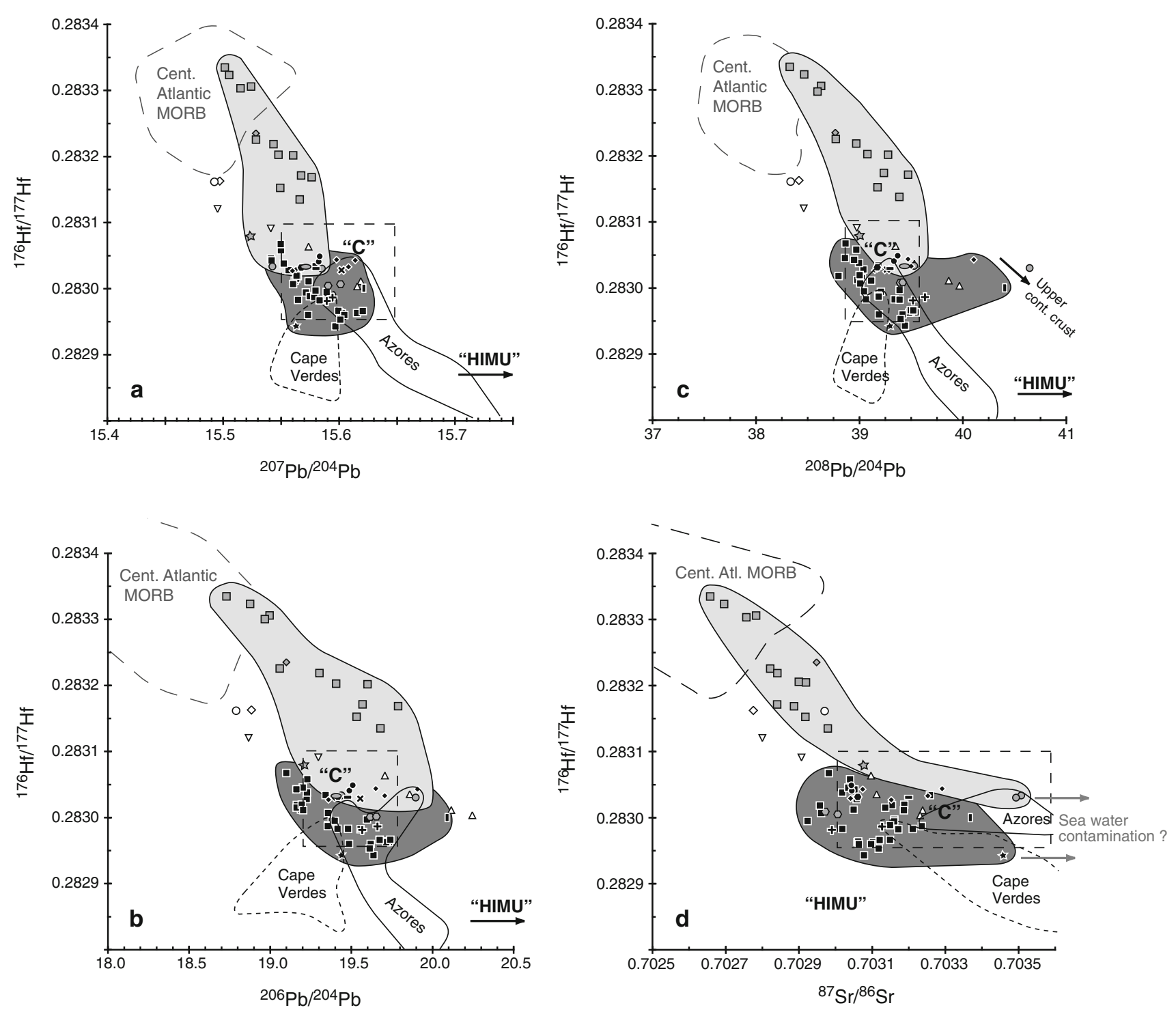

Fig. $4{ }^{176} \mathrm{Hf} /{ }^{177} \mathrm{Hf}$ data from the Madeira and Canary domains and the Madeira-Tore Rise in various isotope spaces. See Fig. 2 for symbols, fields, and additional $\mathrm{Nd}$ and $\mathrm{Pb}$ data sources

sources, Sobolev et al. $(2005,2007)$ developed a method based on $\mathrm{Ni}, \mathrm{Ca}$, and $\mathrm{Mn}$ concentration in olivine phenocrysts. By applying this method to samples from the western Canary Islands, however, it was recently demonstrated that the enriched, HIMU-like isotope signature resides in a peridotitic mantle source lithology, whereas the relatively depleted endmember can be attributed to a pyroxenitic source interpreted as relatively young $(<1 \mathrm{Ga})$ recycled oceanic crust (Gurenko et al. 2009). This relatively young recycling time may preserve both its eclogitic petrology and its MORB-like isotopic composition. In contrast, a longer recycling time ( $>1 \mathrm{Ga}$ ) for the enriched, HIMU-like component would suffice to develop enriched isotopic signatures and to stir its eclogitic component into the peridotitic mantle matrix. As opposed to the Canary samples, olivine phenocryst compositions from enriched Madeira shield stage samples show high Ni concentrations indicating derivation from an enriched pyroxenitic source (Gurenko, personal communication), which is also consistent with observed correlations of whole rock major and trace element compositions with isotope ratios in recent Madeira lavas (Geldmacher et al. 2006b): enriched $\mathrm{Pb}$ and $\mathrm{Nd}$ isotope compositions correlate with elevated $\mathrm{Fe}, \mathrm{K}$, and high $\mathrm{Zr} / \mathrm{Hf}$ and with low $\mathrm{Al}$ concentrations, indicating derivation from eclogitic (pyroxenitic) sources based on high-pressure melting experiments (e.g. Kogiso et al. 1998). Because of this fundamental difference in the inferred source lithology carrying the respective enriched Madeira and Canary domain endmember signals, it is unlikely that the different $\mathrm{Hf}$ isotope ratios reflect simply a 


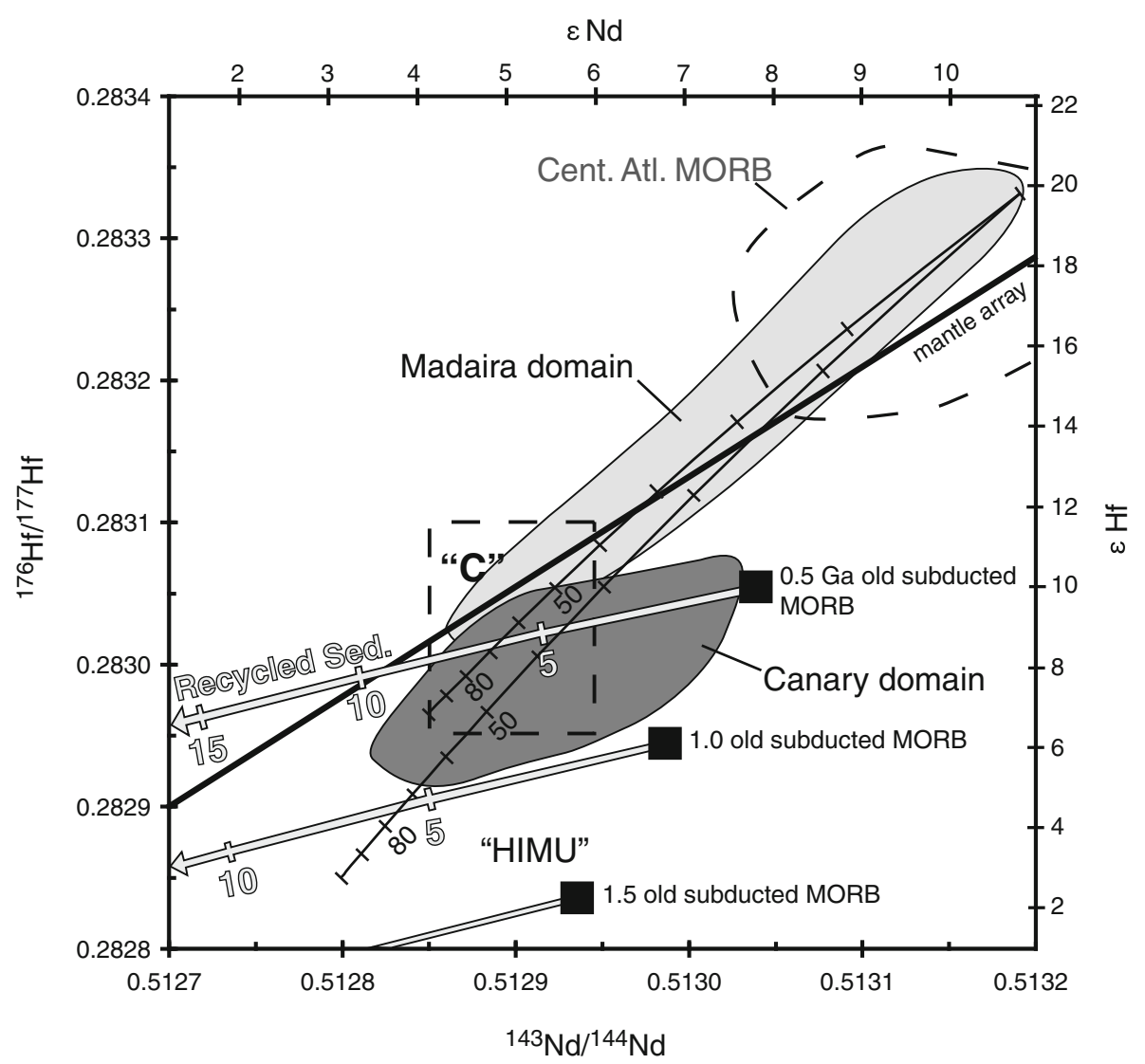

Fig. $5 \mathrm{Nd}$ versus $\mathrm{Hf}$ isotope diagram showing fields for the Madeira and Canary domain (from Fig. 3) compared to present-day compositions of recycled oceanic crust (MORB) of varying ages (black squares) and mixtures of these crusts with associated sediments having the same recycling age (bold gray arrows with tick marks indicating 5\% increments of sediment addition). Modeled MORB crust was extracted at times $0.5,1$, and $1.5 \mathrm{Ga}$ from a mantle source reservoir that was fractionated at $3.9 \mathrm{Ga}$ (main upper mantle fractionation) with parent/daughter ratios of ${ }^{176} \mathrm{Lu} /{ }^{177} \mathrm{Hf}=0.038215$ and ${ }^{147} \mathrm{Sm} /{ }^{114} \mathrm{Nd}=0.214120$. Both values were determined by backcalculating today's bulk silicate Earth's Hf and Nd isotopic compositions to $3.9 \mathrm{Ga}$ (using CHUR Hf isotopic composition of ${ }^{176} \mathrm{Hf} /{ }^{177} \mathrm{Hf}=0.282772$ and ${ }^{176} \mathrm{Lu} /{ }^{177} \mathrm{Hf}=0.0332$ from BlichertToft and Albarède (1997)) yielding ${ }^{176} \mathrm{Hf} /{ }^{177} \mathrm{Hf}=0.280177$ and ${ }^{143} \mathrm{Nd} /{ }^{144} \mathrm{Nd}=0.50756$. These values are used to calculate the initial

quantitatively different contribution of pyroxenitic veins in their mantle sources (e.g., larger/lesser degrees of melting). Rather, these differences suggest contrasting recycling ages.

In contrast to the linear array formed by the Madeira domain, it appears on most isotope plots (particular Figs. 3, and 4) that the Canary domain composition requires a third endmember that seems to overlap (or is identical?) with the enriched Madeira component. Thus, the moderately enriched (low ${ }^{176} \mathrm{Hf} /{ }^{177} \mathrm{Hf}$ ), <1 Ga Madeira pyroxenitic endmember would correspond to the Canary pyroxenite component that was likewise identified by Gurenko et al. (2009) as " $<1$ Ga recycled ocean crust". It is interesting ratios for today's MORB source $\left({ }^{176} \mathrm{Hf} /{ }^{177} \mathrm{Hf}=0.283164\right.$ and ${ }^{143} \mathrm{Nd} /{ }^{144} \mathrm{Nd}=0.513088$ for average zero-age MORB from Chauvel et al. (2008) and $\mathrm{Su}(2002))$ at $0.5,1$ and $1.5 \mathrm{Ga}$, which then evolve to its present-day composition (using today's global average MORB parent/daughter ratios of ${ }^{176} \mathrm{Lu} /{ }^{177} \mathrm{Hf}=0.0270$ and ${ }^{147} \mathrm{Sm} /{ }^{144} \mathrm{Nd}=$ 0.1986, Chauvel et al. (2008)). Numbers and values used to calculate today's composition of subducted sediments (of similar age as the respective subducted crust) from Chauvel et al. (2008) except for the crustal source parent/daughter ratios that were adjusted to the $3.9 \mathrm{Ga}$ crustal fractionation event with ${ }^{176} \mathrm{Lu} /{ }^{177} \mathrm{Hf}=0.03393$ and ${ }^{147} \mathrm{Sm} /{ }^{114} \mathrm{Nd}=0.17897$ (calculated as explained above). Resulting sediment compositions plot to the far left with ${ }^{176} \mathrm{Hf} /{ }^{177} \mathrm{Hf}=$ $0.282637,{ }^{143} \mathrm{Nd} /{ }^{144} \mathrm{Nd}=0.512018(0.5 \mathrm{Ga}) ;{ }^{176} \mathrm{Hf} /{ }^{177} \mathrm{Hf}=0.282445$, ${ }^{143} \mathrm{Nd} /{ }^{144} \mathrm{Nd}=0.511856 \quad(1 \mathrm{Ga})$ and ${ }^{176} \mathrm{Hf} /{ }^{177} \mathrm{Hf}=0.282250$, ${ }^{143} \mathrm{Nd} /{ }^{144} \mathrm{Nd}=0.511693(1.5 \mathrm{Ga})$

that the isotopic composition of this component, common to both the Madeira and Canary domains, also overlaps with the proposed common mantle component "C". The "C" concept, observed in both MORBs and OIBs, is based on linear arrays in $\mathrm{Pb}$ isotope space that converge to a central component that has intermediate $\mathrm{Pb}, \mathrm{Sr}$, and $\mathrm{Nd}$ (and Hf) isotope ratios (Hanan and Graham 1996). "C" is interpreted as relatively young recycled oceanic crust of various ages that has incorporated continental $\mathrm{Pb}$ (e.g. hydrothermal exchange with sediment and/or sea floor alteration) before subduction. Note that the interpretation of "C" differs from the similarly defined FOZO component (FOcus ZOne) that was originally proposed to represent 
relatively undifferentiated, lower mantle (Hart et al. 1992). More recently, Stracke et al. (2005) redefined FOZO as a recycled crust component "formed by continuous subduction and aging of that crust" which is essentially the original "C" interpretation of Hanan and Graham (1996).

The moderately enriched " $\mathrm{C}$ "-like component seems to be not only present in the Madeira and the Canary domains but, based on $\mathrm{Sr}, \mathrm{Nd}$, and $\mathrm{Pb}$ isotope data, also form a possible mixing endmember for the Cape Verde and Azores magma sources (Millet et al. 2009) (Fig. 2). The available Hf isotope ratios from São Miguel and Pico islands in the Azores (Beier et al. 2007; Elliott et al. 2007) and Santiago Island in the Cape Verdes (Barker et al. 2009) seem to support this model (Figs. 3, and 4). On the other hand, such a model appears less obvious on the uranogenic $\mathrm{Pb}$ diagram for the Canary domain (Fig. 2a), unless the linear Canary correlation is considered as being formed by multiple trends between a "C"-like composition and a range of less to more radiogenic $\mathrm{Pb}$ isotope endmembers respectively. If a common " $\mathrm{C}$ " component, derived from recycled oceanic lithosphere, is assumed for all East Atlantic hotspot sources, it may be located within or at a boundary layer at the top of the African superswell to which these hotspots seem to be connected in the tomographic images of Montelli et al. (2006).

Distinct compositional trends for the individual island groups (Madeira, Azores, Cape Verde, and possibly the Canaries) radiating from the intermediate $\mathrm{Pb}, \mathrm{Hf}$, and $\mathrm{Nd}$ isotope space of the "C" component would then represent the unique fingerprint of the individual upwellings (secondary hotspots) ascending from the top of the superswell. For the Madeira domain, this would be a simple mixture with isotopically more depleted material (Geldmacher and Hoernle 2000), while for the Canaries it would be a more complex mixture of the "C" component with a somewhat older, recycled HIMU-like source and a depleted (upper mantle) MORB source-like endmember. According to this model, the HIMU-like source either also resides in the area of the secondary plume origin (above the superswell, dragged up as a surrounding sheath from the source region of the plume; e.g. Lohmann et al. 2009) or gets entrained by the ascending plume material at upper mantle depths (Meyzen et al. 2007). In the latter case, the enriched HIMU-like material could reside in the subcontinental lithospheric mantle.

\section{Conclusions}

1. The Hf isotope data confirm the geochemical distinction between the Canary and Madeira volcanic provinces (domains) in the East Atlantic.
2. Madeira domain Hf isotopic variations can be explained by bimodal mixing between a depleted endmember and a composition located slightly below the Nd-Hf mantle array, modeled as recycled $<1$-Gaold subducted oceanic crust with minor contribution of similarly aged associated sediments.

3. The lower ${ }^{176} \mathrm{Hf} /{ }^{177} \mathrm{Hf}$ ratios of the enriched Canary domain indicate a contribution of material having slightly older recycling ages $(\geq 1 \mathrm{Ga})$.

4. The more widespread Canary domain isotope composition reflects a more complex (heterogeneous) source mixture. The Hf isotope data point toward involvement of a third component that appears to be similar to the Madeira domain enriched endmember. This component has intermediate $\mathrm{Pb}, \mathrm{Hf}$, and $\mathrm{Nd}$ isotope ratios and largely overlaps with the proposed common mantle endmember "C".

5. It is possible that " $\mathrm{C}$ ", seen in both Madeira and possibly Canary domains (and in the nearby Azores and Cape Verde hotspots), reflects material residing at the top or in a boundary layer directly above the large African superswell to which all the secondary East Atlantic hotspots seem to be connected based on tomographic models.

Acknowledgments We thank J. Miller (San Diego), P. Telouk (Lyon), F. Wombacher (Kiel), and S. Hauff (Kiel) for analytical assistance and advice. The paper benefited from the constructive reviews by C. Beier and one anonymous reviewer. Samples from the Madeira Islands, submarine seamounts, and Canary archipelago were obtained with funds from the Deutsche Forschungsgemeinschaft (DFG, German Research Council, grants Ho 1833/1, Ho 1833/9) and the Bundesministerium für Bildung, Wissenschaft, Forschung and Technology (BMBF, Federal Ministry of Education and Research). $\mathrm{BBH}$ and JBT acknowledge financial support from the National Science Foundation (NSF) and the French Institut National des Sciences de l'Univers, respectively. HUS acknowledges support from DFG grant SCHM 250/82-1 and JG from DFG grant GE 1125/1-1.

Open Access This article is distributed under the terms of the Creative Commons Attribution Noncommercial License which permits any noncommercial use, distribution, and reproduction in any medium, provided the original author(s) and source are credited.

\section{References}

Allègre CJ, Turcotte D (1986) Implications of a two-component marble-cake mantle. Nature 323:123-127

Armstrong RL (1968) A model for the evolution of strontium and lead isotopes in a dynamic Earth. Rev Geophys 6:175-199

Anderson DL (1998) The scales of mantle convection. Tectonophysics $284: 1-17$

Anderson DL (2001) Top-down tectonics? Science 293:2016-2018

Anguita F, Hernán F (2000) The Canary Islands origin: a unifying model. J Volc Geotherm Res 103:1-26 
Barker AK, Holm PM, Peate DW, Baker JA (2009) A 5 million year record of compositional variations in mantle sources to magmatism on Santiago, southern Cape Verde archipelago. Contrib Min Petrol. doi:10.1007/s00410-009-0470-x

Becker TW, Boschi L (2002) A comparison of tomographic and geodynamic mantle models. Geochem Geophys Geosys 3:2001GC000168

Beier C, Stracke A, Haase KM (2007) The peculiar geochemical signature of São Miguel lavas: metsamatised or recycled mantle sources? Earth Planet Sci Lett 259(1-2):186-199

Beier C, Haase K, Abouchami W, Krienitz M-S, Hauff F (2008) Magma genesis by rifting of oceanic lithosphere above anomalous mantle: Terceira Rift, Azores. Geochem Geophys Geosys 9. doi:10.1029/2008GC002112

Bernard-Griffiths J, Gruau G, Cornen G, Azambre B, Macé J (1997) Continental lithospheric contribution to alkaline magmatism: isotopic ( $\mathrm{Nd}, \mathrm{Sr}, \mathrm{Pb})$ and geochemical (REE) evidence from Serra de Monchique and Mount Ormonde complexes. J Petrol 38(1):115-132

Blichert-Toft J, Albarède F (1997) The Lu-Hf isotope geochemistry of chondrites and the evolution of the mantle-crust system. Earth Planet Sci Lett 148:243-258

Blichert-Toft J, Chauvel C, Albarede F (1997) Separation of Hf and $\mathrm{Lu}$ for high-precision isotope analyses of rock samples by magnetic sector-multiple collector ICP-MS. Contrib Mineral Petrol 127:248-260

Blichert-Toft J, Frey FA, Albarede F (1999) Hf isotope evidence for pelagic sediments in the source of Hawaiian basalts. Science 285:879-882

Blundy JD, Robinson JAC, Wood BJ (1998) Heavy REE are compatible in clinopyroxene on the spinel lherzolite solidus. Earth Planet Sci Lett 160:493-504

Burke K, Wilson JT (1972) Is the African plate stationary? Nature 239:387-390

Carracedo JC (1994) The Canary Islands: an example of structural control on the growth of large ocean-island volcanoes. J Volc Geotherm Res 60:225-241

Carracedo JC, Day S, Guillou H, Rodriguez-Badiola E, Canas JA, Perrez Torrado FJ (1998) Hotspot volcanism close to a passive continental margin: the Canary Islands. Geol Mag 135:591-604

Chauvel C, Hofmann AW, Vidal P (1992) HIMU-EM: the French Polynesian connection. Earth Planet Sci Lett 110:99-119

Chauvel C, Lewin E, Carpentier M, Arndt NT, Marini J-C (2008) Role of recycled oceanic basalt and sediment in generating the Hf-Nd mantle array. Nature Geosci 1:64-67

Courtillot V, Davaille A, Stock J, Besse J (2003) Three distinct types of hotspots in the Earth's mantle. Earth Planet Sci Lett 205:285-308

Cousens BL, Spera FJ, Tilton GR (1990) Isotopic pattern in silicic ignimbrites and lava flows of the Mogan and Lower Fataga formations, Gran Canaria, Canary Islands: temporal changes in mantle source composition. Earth Planet Sci Lett 96:319-335

D’Oriano F, Angeletti L, Capotondi L, Laurenzi A, Taviani M, Torelli L, Trua T, Zitellini N. (in press) Coral Patch seamount as signature of the Monchique-Madeira hot spot, Eastern Atlantic Sea. Terra Nova

Duncan RA (1984) Age progressive volcanism in the New England Seamounts and the opening of the central Atlantic ocean. J Geophys Res 89(B12):9980-9990

Elliott T, Blichert-Toft J, Heumann A, Koetsier G, Forjaz V (2007) The origin of the enriched mantle beneath São Miguel, Azores. Geochim Cosmochim Acta 71:219-240

Féraud GD, York C, Mével G, Cornen C, Hall M, Auzende J-M (1986) Additional 40Ar-39Ar dating of the basement and the alkaline volcanism of Gorringe Bank (Atlantic Ocean). Earth Planet Sci Lett 79:255-269
Foulger GR, Natland JH (2003) Is "hotspot" volcanism a consequence of plate tectonics. Science 300:921-922

Geldmacher J, Hoernle K (2000) The 72 Ma geochemical evolution of the Madeira hotspot (eastern North Atlantic): recycling of Palaeozoic $(\leq 500 \mathrm{Ma})$ basaltic and gabbroic crust. Earth Planet Sci Lett 183:73-92

Geldmacher J, van den Bogaard P, Hoernle KA, Schmincke H-U (2000) Ar age dating of the Madeira Archipelago and hotspot track (eastern North Atlantic). Geochem Geophys Geosys 1. doi: 10.1029/1999GC000018

Geldmacher J, Hoernle K, van den Bogaard P, Zankl G, GarbeSchönberg D (2001) Earlier history of the $\geq 70$-Ma-old Canary hotspot based on the temporal and geochemical evolution of the Selvagen archipelago and neighboring seamounts in the eastern North Atlantic. J Volc Geotherm Res 111:55-87

Geldmacher J, Hoernle K, van den Bogaard P, Duggen S, Werner R (2005) New ${ }^{40} \mathrm{Ar} /{ }^{39} \mathrm{Ar}$ age and geochemical data from seamounts in the Canary and Madeira volcanic provinces: support for the mantle plume hypothesis. Earth Planet Sci Lett 237:85-101

Geldmacher J, Hoernle K, Klügel A, van den Bogaard P, Wombacher F, Berning B (2006a) Origin and geochemical evolution of the Madeira-Tore Rise (eastern North Atlantic). J Geophys Res 111. doi:10.1029/2005JB003931

Geldmacher J, Hoernle K, Klügel A, van den Bogaard P, Duggen S (2006b) A geochemical transect across a heterogeneous mantle upwelling: implications for the evolution of the Madeira hotspot in space and time. Lithos 90:131-144

Gurenko AA, Hoernle KA, Hauff F, Schmincke H-U, Han D, Miura YN, Kaneoka I (2006) Major, trace element and Nd-Sr-Pb-O$\mathrm{He}-\mathrm{Ar}$ isotope signatures of shield stage lavas from the central and western Canary Islands: insights into mantle and crustal processes. Chem Geol 233:75-112

Gurenko AA, Sobolev AV, Hoernle KA, Hauff F, Schmincke H-U (2009) Enriched, HIMU-type peridotite and depleted recycled pyroxenite in the Canary plume: a mixed-up mantle. Earth Planet Sci Lett 277:514-524

Hanan BB, Graham DW (1996) Lead and helium isotope evidence from oceanic basalts for a common deep source of mantle plumes. Science 272:991-995

Hanan BB, Blichert-Toft J, Kingsley R, Schilling JG (2000) Depleted Iceland mantle plume geochemical signature: artifact or multicomponent mixing? Geochem Geophys Geosys 1. doi: 10.1029/1999GC000009

Hart SR, Hauri EH, Oschmann LA, Whitehead JA (1992) Mantle plumes and entrainment: isotopic evidence. Science 256:517-520

Hirschmann MM, Stolper EM (1996) A possible role for garnet pyroxenite in the origin of the "garnet signature" in MORB. Contrib Mineral Petrol 124:185-208

Hoernle K (1998) Geochemistry of Jurassic ocean crust beneath Gran Canaria (Canary Islands): implications for crustal recycling and assimilation. J Petrol 39(5):859-880

Hoernle K, Schmincke H-U (1993a) The petrology of the tholeiites through melilite nephelinites on Gran Canaria, Canary Islands: crystal fractionation, accumulation and depth of melting. J Petrol 34:573-597

Hoernle K, Schmincke H-U (1993b) The role of partial melting in the 15 Ma geochemical evolution of Gran Canaria: a blob model for the Canary hotspot. J Petrol 34:599-626

Hoernle K, Tilton G (1991) Sr-Nd-Pb isotope data fro Fuerteventura (Canary Islands) basal complex and subaerial volcanics: application to magma genesis and evolution. Schweiz Min Petrograph Mit 71:5-21

Hoernle K, Tilton G, Schmincke H-U (1991) Sr-Nd-Pb isotopic evolution of Gran Canaria: evidence for shallow enriched mantle beneath the Canary Islands. Earth Planet Sci Lett 106:44-63 
Hoernle K, Zhang Y-S, Graham D (1995) Seismic and geochemical evidence for large-scale mantle upwelling beneath the eastern Atlantic and western and central Europe. Nature 374:34-39

Hofmann AW (2003) Sampling mantle heterogeneities through oceanic basalts: isotopes and trace elements. In: Holland HD, Turekian KK (eds) Treatise geochem 2.03. Elsevier, New York, pp 61-101

Hofmann AW, White WM (1982) Mantle plumes from ancient oceanic crust. Earth Planet Sci Lett 57:421-436

Holik JS, Rabinowitz PD, Austin JA (1991) Effects of Canary hotspot volcanism on the structure of oceanic crust off Morocco. J Geophys Res 96(B7):12039-12067

Holm PM, Wilson JR, Christensen BP, Hansen L, Hansen SL, Hein KM, Mortensen AK, Pedersen R, Plesner S, Runge MK (2006) Sampling of the Cape Verde mantle plume: evolution of melt composition on Santo Antao, Cape Verde Islands. J Petrol 47(1):145-189

Kelley KA, Plank T, Farr L, Ludden J, Staudigel H (2005) Subduction cycling of U, Th, and Pb. Earth Planet Sci Lett 234:369-383

King SD, Anderson DL (1998) Edge-driven convection. Earth Planet Sci Lett 160:289-296

King S, Ritsema J (2000) African hot spot volcanism: small-scale convection in the upper mantle beneath cratons. Science 290:1137-1140

Klügel A, Schwarz S, van den Bogaard P, Hoernle KA, WohlgemuthUeberwasser CC, Köster JJ (2009) Structure and evolution of the volcanic rift zone at Ponta de São Lourenco, eastern Madeira. Bull Volc 71:671-685

Kogiso T, Hirose K, Takahashi E (1998) Melting experiments on homogenous mixtures of peridotite and basalt. Application to the genesis of ocean island basalts. Earth Planet Sci Lett 216:603-617

Lohmann FC, Hort M, Phipps Morgan J (2009) Flood basalts and ocean island basalts: a deep source or shallow entrainment? Earth Planet Sci Lett 284:553-563

Martins S, Mata J, Munhá Mendes AH, Maerschalk C, Caldeira R, Mattielli N (2010) Chemical and mineralogical evidence of the occurrence of mantle metasomatism by carbonate-rich melts in an oceanic environment (Sabtiago Island, Cape Verde). Min Petrol 99:43-65

Mata J, Kerrich R, MacRae ND, Wu TW (1998) Elemental and isotopic $(\mathrm{Sr}, \mathrm{Nd}$, and $\mathrm{Pb}$ ) characteristics of Madeira Island basalts: evidence for a composite HIMU-EM I plume fertilizing lithosphere. Can J Earth Sci 35:980-997

Merle R, Schärer U, Girardeau J, Cornen G (2006) Cretaceous seamounts along the continent-ocean transition of the Iberian margin: $\mathrm{U}-\mathrm{Pb}$ ages and $\mathrm{Pb}-\mathrm{Sr}-\mathrm{Hf}$ isotopes. Geochim Cosmochim Acta 70(19):4950-4976

Merle R, Jourdan F, Marzoli A, Renne PR, Grange M, Girardeau J (2009) Evidence of multi-phase Cretaceous to Quaternary alkaline magmatism on Tore-Madeira Rise and neighbouring seamounts from ${ }^{40} \mathrm{Ar} /{ }^{39} \mathrm{Ar}$ ages. J Geol Soc London 166:879-894

Meyzen CM, Blichert-Toft J, Ludden JN, Humler E, Mével C, Albarède F (2007) Isotopic portrayal of the Earth's upper mantle flow field. Nature 447:1069-1074

Millet AA, Doucelance R, Baker JA, Schiano P (2009) Reconsidering the origins of isotopic variations in ocean island basalts; insights from fine-scale study of São Jorge Island, Azores archipelago. Chem Geol 265:289-303

Montelli R, Nolet G, Dahlen FA, Masters G (2006) A catalogue of deep mantle plumes: new results from finite frequency tomography. Geochem Geophys Geosys 7:Q11007. doi: 10.1029/2006GC001248

Morgan WJ (1972) Plate motions and deep mantle convection. Geol Soc Am Mem 132:7-22
Ni SD, Ding XM, Helmberger DV, Gurnis M (1999) Low velocity structure beneath Africa from modeling. Earth Planet Sci Lett 170(4):497-507

Peirce C, Barton PJ (1991) Crustal structure of the Madeira-Tore Rise, eastern North Atlantic-results of a DOBS wide-angle and normal incidence seismic experiment in the Josephine Seamount region. Geophys J Int 106:357-378

Plank T, Langmuir CH (1998) The chemical composition of subducting sediment and its consequences for the crust and mantle. Chem Geol 145:325-394

Rickli J, Frank M, Halliday AN (2009) The hafnium-neodymium isotopic composition of Atlantic seawater. Earth Planet Sci Lett 280:118-127

Ritsema HJ, van Heijst JH, Woodhouse JH (1999) Complex shear velocity structure beneath Africa and Iceland. Science 286:1925-1928

Salters VJM, Hart SR (1989) The Hf-paradox, and the role of garnet in the MORB source. Nature 342:420-422

Salters VJM, White WM (1998) Hf isotope constraints on mantle evolution. Chem Geol 145:447-460

Schilling JG (1975) Azores mantle blob: rare-earth evidence. Earth Planet Sci Lett 25:103-115

Schilling JG, Thompson G, Kingsley R, Humphris S (1985) Hotspotmigrating ridge interaction in the South Atlantic. Nature 313:187-191

Schmincke H-U (1982) Volcanic and chemical evolution of the Canary Islands. In: Seibold E et al (eds) Geology of the northwest African margin. Springer, New York, pp 273-306

Schmincke H-U, Sumita M (2010) Geological evolution of the Canary Islands. Görres Verlag, Koblenz, pp 1-196

Sleep NH (2002) Ridge-crossing mantle plumes and gaps in tracks. Geochem Geophys Geosys 3(12). doi:10.1029/2001GC000290

Smith WHF, Sandwell DT (1997) Global seafloor topography from satellite altimetry and ship depth soundings. Science 277:1956-1962

Sobolev AV, Hofmann AW, Sobolev SV, Nikogosian IK (2005) An olivine-free mantle source of Hawaiian shield basalts. Nature 434:590-597

Sobolev AV, Hofmann AW, Kuzmin DV, Yaxley GM, Arndt NT, Chung S-L, Danyushevsky LV, Elliott T, Frey FA, Garcia MO, Gurenko AA, Kamenetsky VS, Kerr AC, Krivolutskaya NA, Matvienkov VV, Nikogosian IK, Rocholl A, Sigurdsson IA, Sushchevskaya NM, Teklay M (2007) The amount of recycled crust in sources of mantle-derived melts. Science 316:412-417

Stalder R, Foley SF, Brey GP, Horn I (1998) Mineral-aqueous fluid partitioning of trace elements at $900-1200^{\circ} \mathrm{C}$ and $3.0-5.7 \mathrm{GPa}$ : new experimental data for garnet, clinopyroxene, and rutile, and implications for mantle metasomatism. Geochim Cosmochim Acta 62:1781-1801

Stracke A, Bizimis M, Salters VJM (2003) Recycling oceanic crust: quantitative constraints. Geochem Geophys Geosys 4 (3). doi: 10.1029/2001GC000223

Stracke A, Hofmann AW, Hart SR (2005) FOZO, HIMU, and the rest of the mantle zoo. Geochem Geophys Geosys 6(5). doi: $10.1029 / 2004 G C 000824$

$\mathrm{Su}$ YJ (2002) Mid-ocean ridge basalt trace element systematics: constraints from database management, ICP-MS analyses, global data compilation and petrological modeling. Dissertation, Columbia University

Suetsugu D, Isse T, Tanaka S, Obayashi M, Shiobara H, Sugioka H, Kanazawa T, Fukao Y, Barruol G, Reymond D (2009) South Pacific mantle plumes imaged by seismic observation on islands and seafloor. Geochem Geophys Geosys 10. doi:10.1029/ 2009GC002533 
Thompson PME, Kempton PD, Kerr AC (2008) Evaluation of the effects of alteration and leaching on Sm-Nd and Lu-Hf systematics in submarine mafic rocks. Lithos 104(1):164-176

Todt W, Cliff RA, Hanser A, Hofmann AW (1996) Evaluation of a ${ }^{202} \mathrm{~Pb}^{205} \mathrm{~Pb}$ double spike for high precision lead isotope analyses. In: Basu A, Hart S (eds) Earth processes: reading the isotopic code. Geophys Monogr 95, AGU, Washington, pp 429-437

Torsvik TH, Smethurst MA, Burke K, Steinberger B (2006) Large igneous provinces generated from the margins of the large lowvelocity provinces in the deep mantle. Geophys J Int 167:1447-1460

Vervoort JD, Patchet JP, Blichert-Toft J, Albarède F (1999) Relationships between Lu-Hf and Sm-Nd isotope system in the global sedimentary system. Earth Planet Sci Lett 168:79-99

White WM, Schilling J-G, Hart SR (1976) Evidence for the Azores mantle plume from the strontium isotope geochemistry of the Central North Atlantic. Nature 263:659-663

Widom E, Hoernle KA, Shirey SB, Schmincke H-U (1999) Os isotope systematics in the Canary Islands and Madeira: lithospheric contamination and mantle plume signature. J Petrol 40(2):279-296

Zhao D (2003) Global tomographic images of mantle plumes and subducting slabs: insight into deep Earth dynamics. Phys Earth Planet Inter 146:3-34

Zindler A, Hart SR (1986) Chemical geodynamics. Annu Rev Earth Planet Sci 14:493-571

Zindler A, Jagoutz E, Goldstein S (1982) Nd, Sr and Pb isotopic systematics in a three component mantle: a new perspective. Nature 298:519-523

Zindler A, Staudigel H, Batiza R (1984) Isotope and trace element geochemistry of young Pacific seamounts: implications for the scale of upper mantle heterogeneity. Earth Planet Sci Lett 70:175-195

Zitellini N, Gràcia E, Matias L, Terrinha P, Abreu MA, DeAlteriis G, Henriet JP, Dañobeitia JJ, Masson DG, Mulder T, Ramella R, Somoza L, Diez S (2009) The quest for the Africa-Eurasia plate boundary west of the Strait of Gibraltar. Earth Planet Sci Lett 280:13-50 